Document downloaded from:

http://hdl.handle.net/10251/83004

This paper must be cited as:

Castellanos Hernández, WE.; Guerri Cebollada, JC.; Arce Vila, P. (2016). A QoS-aware routing protocol with adaptive feedback scheme for video streaming for mobile networks. Computer Communications. 77:10-25. doi:10.1016/j.comcom.2015.08.012.

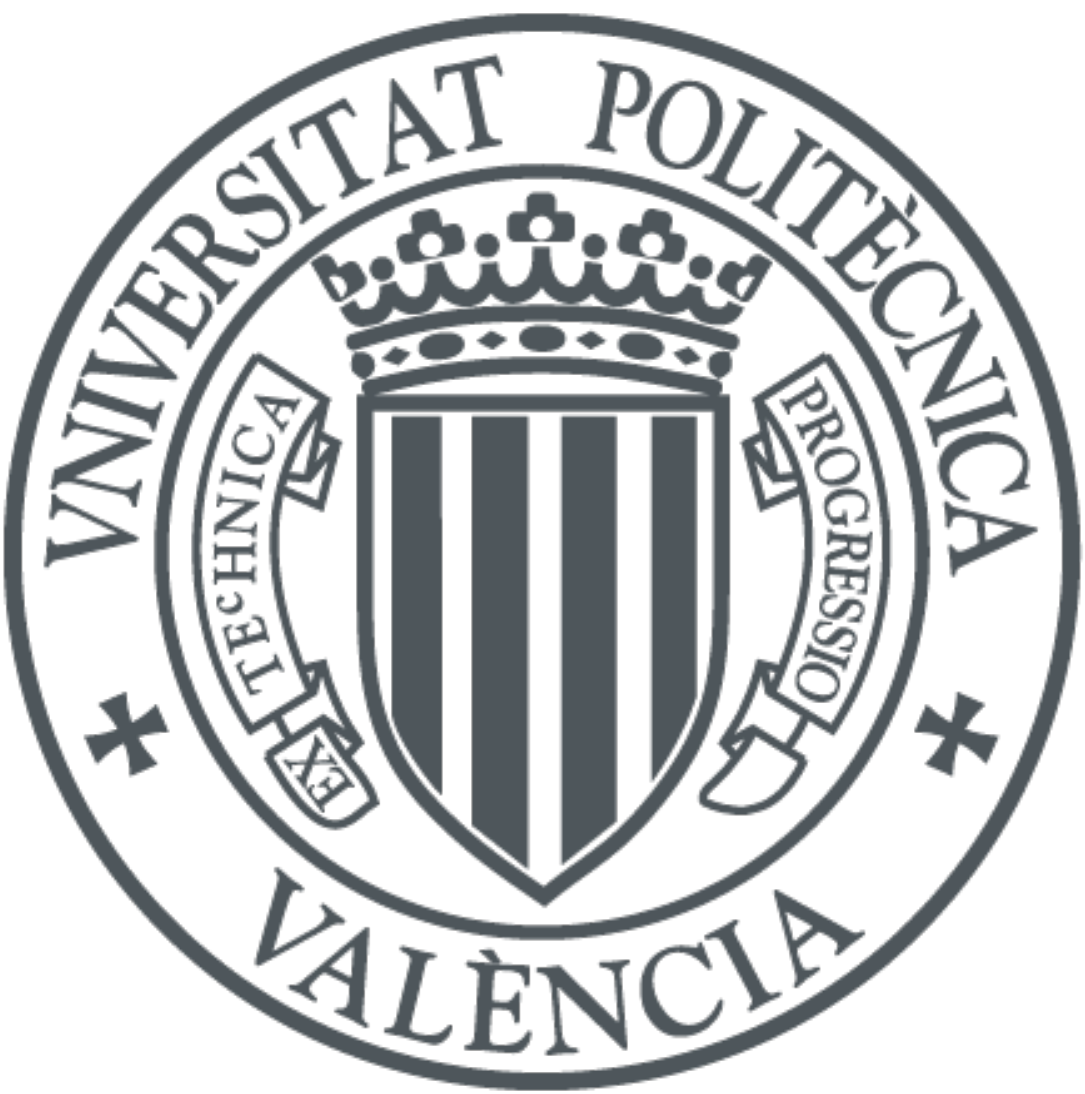

The final publication is available at

http://dx.doi.org/10.1016/j.comcom.2015.08.012

Copyright Elsevier

Additional Information 


\title{
A QoS-aware routing protocol with adaptive feedback scheme for video streaming for mobile networks
}

\author{
Wilder E. Castellanos, Juan C. Guerri and Pau Arce \\ Institute of Telecommunications and Multimedia Applications (iTEAM), Universitat Politècnica de València, Camino de Vera, 46071, Valencia, \\ Spain. \\ E-mail: wilcashe@upv.es, jcguerri@dcom.upv.es, paarvi@iteam.upv.es
}

\begin{abstract}
One of the major challenges for the transmission of time-sensitive data like video over Mobile Ad-Hoc Networks (MANETs) is the deployment of an end-to-end QoS support mechanism. Therefore, several approaches and enhancements have been proposed concerning the routing protocols. In this paper we propose a new QoS routing protocol based on AODV (named AQA-AODV), which creates routes according to application QoS requirements. We have introduced link and path available bandwidth estimation mechanisms and an adaptive scheme that can provide feedback to the source node about the current network state, to allow the application to appropriately adjust the transmission rate. In the same way, we propose a route recovery approach into the AQA-AODV protocol, which provides a mechanism to detect the link failures in a route and re-establish the connections taking into account the conditions of QoS that have been established during the previous route discovery phase. The simulation results reveal performance improvements in terms of packet delay, number of link failures and connection setup latency while we make more efficient use of the available bandwidth than other protocols like AODV and QAODV. In terms of video transmission, the obtained results prove that the combined use of AQA-AODV and the scalable video coding provides an efficient platform for supporting rate-adaptive video streaming.
\end{abstract}

\section{KEYWORDS}

Wireless ad hoc networks, QoS routing, Adaptive Video Streaming, Video transmission over MANETs, AODV, QoS AODV.

\section{INTRODUCTION}

A Mobile Ad-doc Network (MANET) consists of a collection of mobile nodes that communicate in a multi-hop way without a fixed infrastructure. MANETs are very versatile and appropriate to be used in many scenarios due to the infrastructure-less and self-organized characteristics. However, they have different limitations such as bandwidthconstrained, variable capacity links and energy-constrained operation. Moreover, routes may include multiple hops because communications need to use intermediate nodes as routers in order to communicate with nodes that are out of its transmission range. This dynamic topology of nodes causes frequent link failures and high error rates, so it makes it difficult to maintain the desired Quality of Service (QoS) in the network. Additionally, due to the fact that the wireless channel is shared among neighbour nodes and that network topology can change as nodes move, the transmission of time-sensitive data (e.g. video packets) is made more difficult [1]. Furthermore, with the prevalence of multimedia applications, it has become very necessary for MANETs to have an efficient routing and QoS mechanisms to support these applications. Thus, traditional best-effort protocols are not adequate. This is because multimedia applications require the underlying network to provide certain guarantees that are manifested in the support of several important QoS parameters such as bandwidth, delay, jitter and packet loss rate.

We propose in this paper a cross-layer strategy for adaptive video streaming in MANETs based on the estimation of the available network resources and the subsequent adaptation of the transmission rate. The main contribution of this work is the development of a comprehensive QoS routing protocol, named AQA-AODV (Adaptive QoS-Aware for Ad hoc On-demand Distance Vector). Our approach include novelty features In addition, we propose the use of AQAAODV in conjunction with the Scalable Video Coding (H.264/SVC) [2] as a realistic solution for supporting rateadaptive video streaming.

AQA-AODV is a modified and enhanced version of the routing protocol AODV (Ad hoc On-demand Distance Vector) [3]. More precisely, we have introduced into the original AODV protocol an adaptive feedback scheme and two mechanisms: one for the estimation of the available bandwidth in each node and the other for the prediction of the consumed bandwidth for a route of multi-hops. In addition, some QoS fields are added to the AODV control packets and the routing table. The Generalized MANET packet/message format [4] has been considered in the definition of the routing messages of AQA-AODV. Therefore, although our protocol has been designed as an enhancement of 
AODV, the proposed algorithms and the new packet fields can be integrated into AODVv2 [5] in order to provide QoS mechanisms to this routing protocol.

On the other hand, Scalable Video Coding is a flexible coding technique where the video streams are composed of a base layer and one or more enhancement layers, which may enhance the spatio-temporal resolution and/or quality of the base layer. Based on such scalable-layered structure, a video stream can be easily adapted to meet constraints imposed by devices and networks adding or removing SVC layers. For an effective SVC adaptation, AQA-AODV provides a cross layer approach in order to estimate the available bandwidth. Such information is later sent to the video application to adjust the amount of layers that can be transmitted. This network-adaptive strategy avoids congestion and a large number of dropped packets. Congestion and losses are worse than transmitting video using low data rate. This design concept is consistent with the current paradigm, known as application-oriented paradigm, which involves a new strategy of development of solutions for MANETs where application requirements are identified before the development of the technical solutions [6].

We conducted a performance evaluation of our proposed solution in order to demonstrate that it is an effective system for providing video streaming services over MANETs. In particular, the evaluation focuses on the analysis of traffic metrics, such as packet losses and end-to-end delay as well as metrics specifically related to video quality (such as PSNR and decoded frame rate). We have developed a novel simulation framework (named SVCEval-RA [7]) to perform the simulation experiments, which represents an additional contribution of this paper. This software tool integrates the network simulator NS-2 [8] with external tools for analysing H.264/SVC video streams. Our framework provides an efficient platform in order to perform simulation studies that involve rate-adaptive video streaming. The experimental results show that the combined use of AQA-AODV and scalable video coding provides an efficient system for supporting adaptive video streaming where video application can adapt its bit rate according to the available bandwidth. Consequently, the quality of the received videos has been significantly improved.

The rest of the paper is organized as follows. First, we introduce related works on QoS routing for MANETs in Section 2. Then, in Section 3 we describe the impact of the channel capacity and the packet forwarding over delay and packet loss in wireless ad hoc networks. In addition, we briefly review the main characteristics of AODV and QAODV protocols. In Section 4 we present a more detailed explanation of the main components of AQA-AODV protocol. Section 5 gives a brief introduction to the scalable video coding. The results of the performance evaluation of the proposed QoS-aware routing protocol are described in Section 6 and finally, we present our conclusions in Section 7.

\section{RELATED WORK}

Video transmission over wireless ad hoc networks has been discussed during last years and it has become an attractive topic in many papers and research works. However, actually the provision of video streaming services over MANETs is still a challenging task due to the difficulty of meeting certain levels of QoS. Hence, several approaches have been proposed to provide QoS in mobile ad hoc networks, which can be classified according to the layer they operate. Some recent approaches for providing QoS in MAC layer can be consulted in references [9-12] and in the survey [13]. Regarding the QoS solutions for network layer, most of the QoS routing protocols are the extensions of existing besteffort routing protocols. Numerous reactive and proactive QoS routing protocols have been proposed for MANETs recently. Nevertheless, in this paper focus is on reactive QoS routing protocols and mainly those solutions based on the well-known AODV routing protocol. For example, Su et al. in [14] and Zhen \& Wenzhong in [15] proposed some approaches which use AODV as routing protocol within a TDMA (Time Division Multiple Access) network. However, TDMA has a less efficient controlled access scheme because of the lack of infrastructure and the peer-topeer nature of ad hoc networks. Other QoS routing protocols are based on the Internet draft [16] (called QAODV) which describes the format and extensions to provide QoS support in AODV. Some approaches of this kind are described in references [17], [18], [19], [20] and [21]. They are based on the model of admission control of QAODV without any mechanism of feedback. Therefore, the source cannot know the available resources of the network. Moreover, the initial QoS conditions are not maintained after link failures due to the lack of a suitable route recovery algorithm. Other solution based on AODV is the RBRP protocol proposed by Tabatabaei et al. [22]. They extend the route discovery process using the Q-learning strategy to select a stable route to enhance network performance. This technique improves performance achieved with AODV through an enhanced route selection based on hop count, bandwidth, power of battery and speed of mobile nodes. However, this proposal does not improve the performance achieved by other QoS routing solutions because of it does not take into consideration some constraints inherent in the mobile ad hoc networks (e.g. the mutual interference of the nodes). This fact leads an inaccuracy estimation of the available bandwidth. On the other hand, Quin et al. [23] proposed a solution called ORAC, where a cooperative communication strategy (opportunistic routing) and an admission control scheme are integrated to provide certain QoS for different types of multiple flows. Despite this approach achieves improvements in terms of throughput, average delay and energy consumption, its performance is significantly degraded in mobile scenarios. 
Although numerous research works have been mainly focused on the network layer, video delivery can be improved through cross-layer techniques since some functions cannot be assigned to a single layer. In this sense new solutions involving several abstraction layers have been proposed [24-27]. Hence, it is worth considering cross-layer routing solutions, which can extract useful information from other networks layers. For instance, video awareness could offer new mechanisms to improve video transmissions, such as bandwidth adaptation, intra-frame prioritization or even algorithms that react to the play-out buffer state, obviously at the expense of adding complexity. This contentawareness leads to other solutions based on enhanced video coding. These solutions can support adaptive video streaming schemes using versatile techniques, such as Scalable Video Coding (SVC) [28,29] or Multi-description Coding (MDC) [30,31]. In fact, cross-layer solutions can provide enough information to upper layers in order to adapt video rate accordingly increasing the quality of video streaming services while the bandwidth efficiency is achieved. Despite the complexity of providing hard QoS for multimedia applications over MANETs, there are still many options to improve video streaming quality, through holistic approaches that involve routing, transport and application layers.

\section{BACKGROUND}

Providing quality of service support for wireless ad hoc networks is very challenging, due to many factors, e.g. the use of a shared communication medium. Difficulties lie in the limitation of the maximum achievable throughput caused by the fact that nodes cannot simultaneously access the medium. More specifically, when a node is transmitting a packet, neighbour nodes within its Interference Range (IR), have to keep silent. This fact degrades the wireless data rate. Even more, when a transmission is established, the nodes must cooperate to forward the packets through the network, which means that the available throughput on each host is limited not only by the access channel, but also by the forwarding load. Therefore, network performance is highly impacted causing considerable packet losses and higher delays. Li et al. presented in [32] a more detailed study about the capacity of wireless ad hoc networks. The results of Li et al. suggest that capacity along a route can be surprisingly low. The maximum throughput of one flow is decreased substantially due to the overhead of MAC layer and the mutual interference between packets of the same flow, also called "Intra-flow contention" [33]. Therefore, the packet losses and end-to-end delay are significantly increased, both important metrics for video transmission over wireless ad hoc networks.

AODV and QAODV are important references to contrast the performance of our routing protocol as presented in Section 4 . Therefore, their main characteristics are summarized as follows.

AODV [3] is a widely accepted routing protocol for MANETs which uses a broadcast route discovery mechanism. When a source needs a route to a destination, it broadcasts a Route Request (RREQ) packet in search of a route. A node after receiving an $R R E Q$ sends a Route Reply (RREP) packet to the source, if it either is the destination node, or has an active (fresh) route to the destination. Otherwise, it rebroadcasts the $R R E Q$ packet to its neighbours and creates a reverse route entry for the source. An intermediate node receiving $R R E P$ packet creates a forward route entry for the destination and further forwards the RREP packet toward the source using its reverse route entry. Finally, when source receives multiple $R R E P S$, it selects the $R R E P$ with the highest destination sequence number. Sequence numbers are used to ensure fresh and loop-free routes.

To provide quality of service, extensions can be made in the routing table and in the packets used during the route discovery process. As described in section 2, there are several QoS routing solutions based on AODV. The most important approach is called QAODV which is defined in the internet draft [16]. However, this proposal does not specify how some elements of the routing protocol must be implanted, such as: the methods to compute available bandwidth and the end-to-end delay, the route recovery process due to link failure and the admission control mechanism. Due to these shortcomings, QAODV does not show acceptable results during overload network condition. Hence, some traffic flows can be rejected on the basis that they cannot be carried. However, maybe the source application could adjust some parameters in the coding to adapt its data rate to the network condition. The feedback scheme implemented in our AQA-AODV model indicates to the source node about the status of the network and makes more efficient use of the available bandwidth. Moreover, a mechanism for the estimation of the available bandwidth and the route recovery process are defined.

\section{QOS-AWARE AODV PROTOCOL WITH ADAPTIVE FEEDBACK SCHEME}

In this section, we describe the details of our proposed routing protocol called AQA-AODV (Adaptive QoS-Aware Ad-hoc On-demand Distance Vector), which is a protocol based on AODV. Our key modifications affect the route discovery phase and the route maintenance strategy of AODV. These modifications are:

i) An algorithm used for the estimation of the available bandwidth that allows nodes along the path to know their available resources (in terms of bandwidth). 
ii) A cross-layer mechanism to inform to the application layer the available bandwidth by which the source node can easily adapt its transmission rate.

iii) A route recovery mechanism with a session cache table.

Some changes in the format of the packets used in AODV are required to implement the above modifications. For example, we added a QoS extension with new fields to the $R R E Q$ and $R R E P$ packets to carry the information about bandwidth requirements, transmission rate and a session ID (used to identify each QoS flow). The new AQA-AODV packets are called $Q R R E Q$ and $Q R R E P$. In addition, the packet formats have been updated according to the Generalized MANET packet/message format [4].

An important difference between our proposed protocol and other solutions based on AODV is the adaptive feedback scheme, integrated into the routing protocol, by which the source node can know and easily adapt its transmission rate according to the state of the route. For this reason, nodes along the path must know their available resources by using some algorithms.

Fig. 1 depicts the functional block diagram of AQA-AODV. The main three elements of AQA-AODV are a bandwidth estimation module, a routing module and a route recovery module. The first module performs the estimation of the available bandwidth and provides data feedback to the video application. HELLO packets are used in the bandwidth estimation, which is periodically executed according to the trigger of Timer module. The information about the available bandwidth is used by video application in order to compose a video stream extracting the layers, from the SVC video stream, that cannot be supported by network. On the other hand, the routing module receives the route requests from the application and executes the route discovery routine. When a route between source and destination is established, a unique session identifier ( sid) is assigned in the Session/sid mapper. The identification data of the sessions ( $\mathrm{sid}$, source and destination address, QoS requirements and expiration time) are stored internally in a database, called Session Cache List. The third main module is the route recovery module, which is in charge of re-establishing the connections after a link failure, taking into account the QoS conditions of each of the sessions.

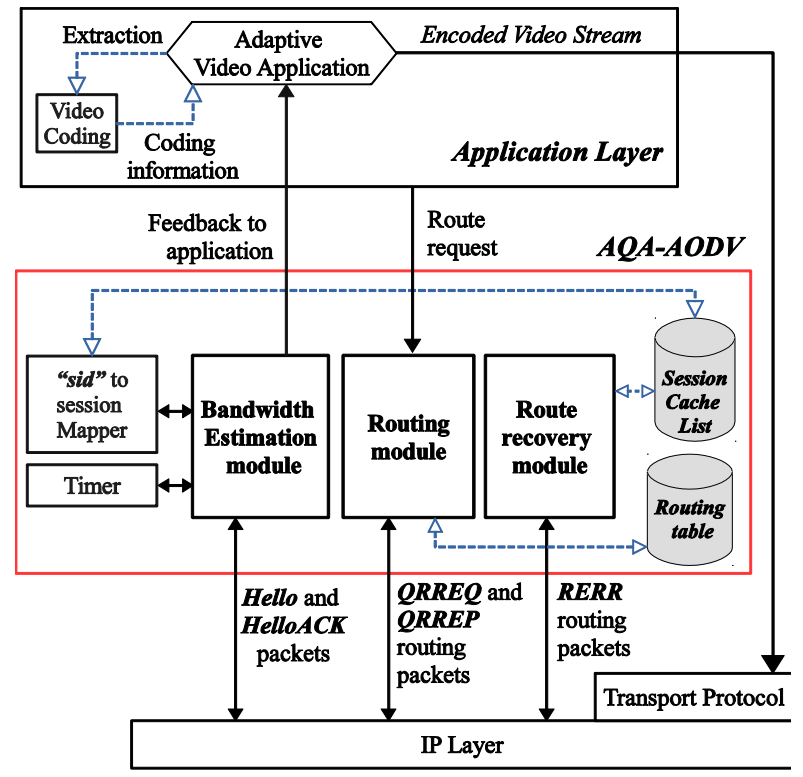

Fig. 1. Functional block diagram of $A Q A-A O D V$

In next sub-sections, we describe the main tasks performed by AQA-AODV. First, we describe the algorithms used in the bandwidth estimation phase. Then, we give a more detailed explanation of the routines involved in the route discovery phase as well as the mechanisms of the route recovery strategy.

\subsection{Bandwidth Estimation Phase}

When an incoming flow is requesting admission in a wireless ad hoc network, the optimum transmission rate must be estimated in order to be informed to the source node. The optimum transmission rate is the data rate at which a source node sends packets achieving the highest throughput without causing congestion in the network. Therefore, this rate must be equal to or less than the available end-to-end bandwidth from the source to the destination. In wired networks, the available end-to-end bandwidth is a concave parameter, which is determined by the minimum available bandwidth of the links along the route (bottleneck bandwidth). However, this is still a challenging problem in wireless ad hoc networks due to many factors such as the shared nature of the wireless channel and the mobility. Moreover, a packet 
emission from a node has an impact on the available bandwidth of nodes located in a certain area surrounding the source node. This causes a decrease of the data rate that can support each node.

As an example, we show in Fig. 2 a wireless ad hoc network where the available bandwidth (in Kbps) of each link is known. We assume that the source node requests to send data with a rate of $120 \mathrm{Kbps}$ to node 6 . According to the values of the available bandwidth along the path, we assume that the source node adapts its transmission rate to 100 Kbps, which correspond to the bottleneck bandwidth. However, during transmission the maximum throughput reached in the destination node is lower than the expected value. This is caused by the mutual interference between packets of the same flow (also called "intra-flow contention"). Intra-flow contention occurs when nodes along a multihop route contend among themselves for channel access to forward packets belonging to the same flow.

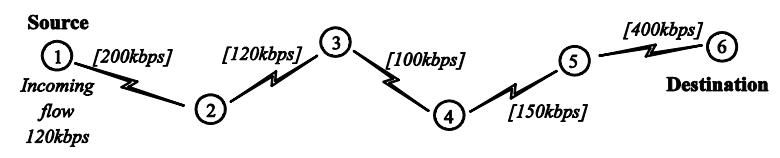

Fig. 2. Example of wireless ad hoc network with the available bandwidth of each link

According to the previous example, two different stages can be identified in the process of feedback of the optimum data rate to the source node: $i$ ) the local estimation of each node of its available bandwidth (BWav) in order to know the available end-to-end bandwidth along the route, and $i$ ) the prediction of the total amount of bandwidth that the new flow can consume ( $B W_{\text {consumed }}$ ) along a route of $n$ hops taking into account the "intraflow contention". Therefore, we propose a new evaluation method of the available end-to-end bandwidth. Our method includes performing checks on all nodes along the route in order to verify if each node could support the consumed bandwidth $\left(B W_{\text {consumed }}\right)$ according to his local estimation of the available bandwidth.

The diagram shown in the Fig. 3 describes in general form, the main tasks involved in the admission of incoming flows. The routine $P 1$ is performed in each node using only its local perception and $P 2$ is executed by destination node based on the requested rate. From the local available bandwidth estimated locally in the nodes, it is determined the available end-to-end bandwidth (P3). The criteria used for accepting a new flow is shown in the decision block D1. That is, if consumed bandwidth by the incoming flow is lower than the available end-to-end bandwidth, then the source node can to transmit at the requested data rate. Otherwise, the source node must adapt its data rate to the value of the available end-to-end bandwidth measured in the path. In order to ensure that all nodes along the route could support the new flow, the condition $D 1$ must be checked in the intermediate nodes from source to destination node.

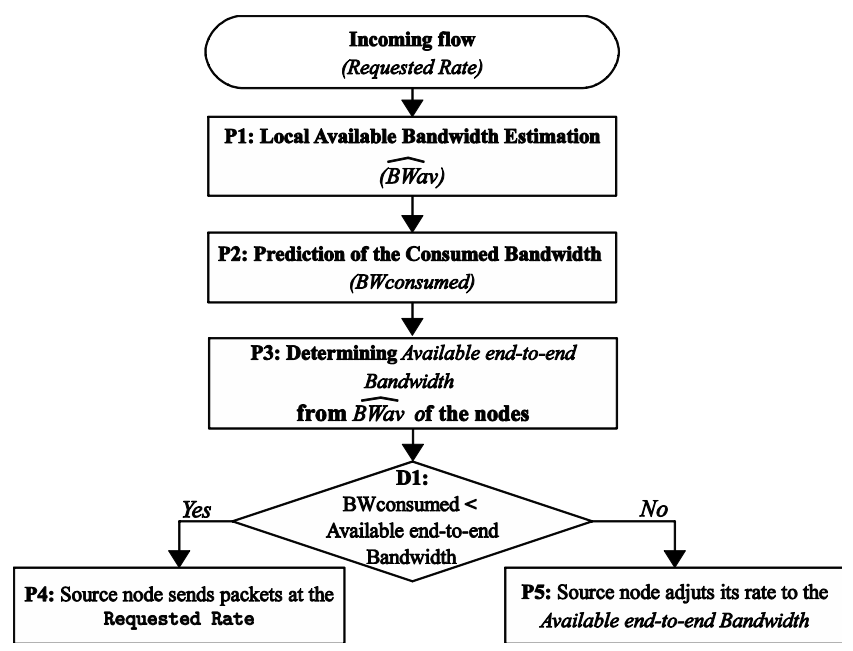

Fig. 3. Flowchart of the tasks involved in the bandwidth estimation phase

In next subsections, we provide a brief description about the algorithms used to estimate the Local Available Bandwidth $\left(B W_{a v}\right)$ in each node and to predict the bandwidth to be consumed ( $\left.B W_{\text {consumed }}\right)$ by the requesting flow.

\subsubsection{Estimation of Local Available Bandwidth in AQA-AODV}

Our proposed algorithm for estimating local available bandwidth consists of two steps. In the first step, each node estimates its local available bandwidth and, in the second one, the nodes calculate a weighted average of the most recent values in order to obtain a final estimation of the local available bandwidth. 
In the first step, we assume that the local available bandwidth between two nodes is defined as the maximum throughput that can be transmitted between these two nodes without negatively affecting any ongoing flow in the network (permissible throughput). The measured throughput allows the node to infer the bandwidth that it has available $(B W a v)$ to transmit a new traffic flow. In our approach, a given node can estimate its permissible throughput to each neighbour by the Equation 1 . Where $S$ is the size (in terms of bits) of all packets sent from one node to its neighbour during the period $T$, where $T$ is equal to $T r-T s$.

$$
T h_{\text {packet }}=\frac{S}{T r-T S}
$$

We propose the measurement of the parameters of the Equation 1 by using HELLO packets, which are used to discover neighbours in AODV. However, an additional packet must be created. We have called this packet HelloACK. In our implementation, the timestamp $T s$ indicates when HELLO packet was sent from sender and $\operatorname{Tr}$ is the time when the HelloACK is received by the sender. Fig.4 illustrates how the HELLO and HelloACK packets are used in the estimation of the permissible throughput. The implementation of our algorithm can be explained with more details as follows. Let $i$ be a node and $j$ its neighbour. To estimate the permissible throughput at the node $i$, a HELLO packet is sent from node $i$ to $j$ (Fig.4(a)). The time-stamp $(T s)$ when the packet is ready to be sent is recorded. When node $j$ receives the HELLO packet, it sends back to node $i$ a HELLOAck packet carrying the time-stamp $T s$. Finally, the time-stamp $T r$ is recorded in node $i$ when the HELLOAck packet is received (Fig.4(b)). The additional HELLOAck packet make more accurate the measurement of the throughput since this measurement will not depend of the throughput seen by only one packet.

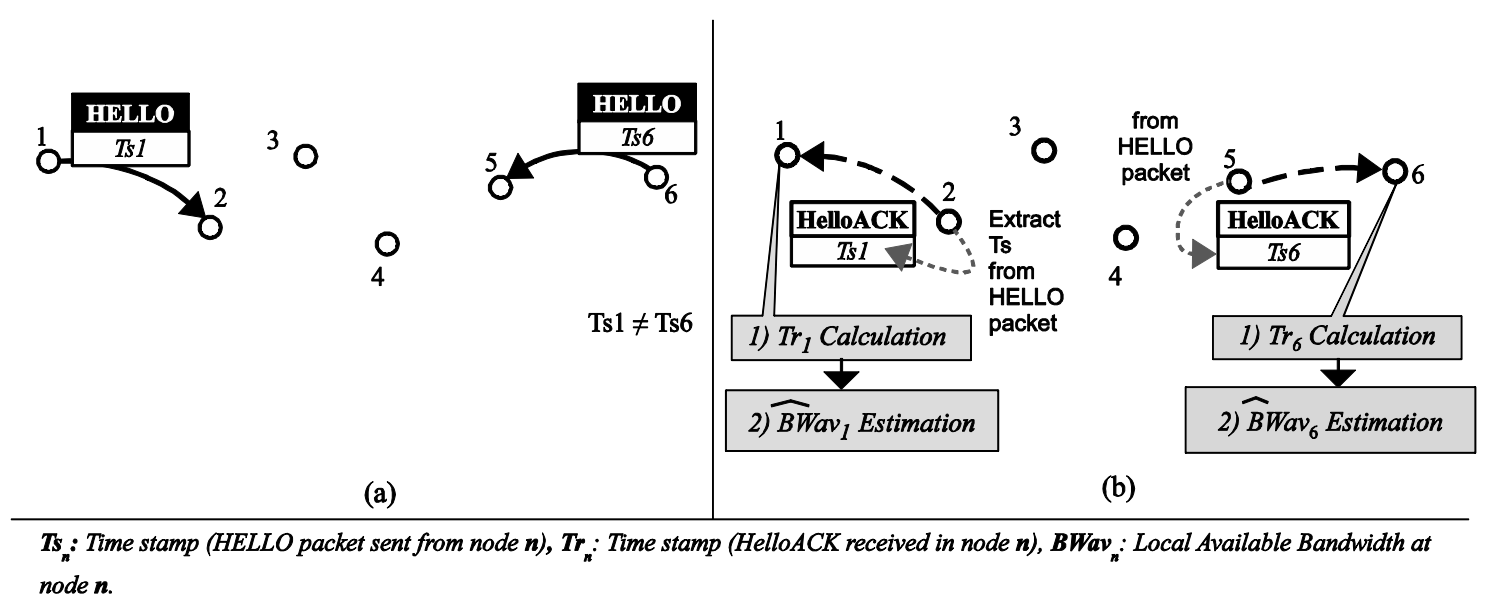

Fig. 4. Using HELLOs packets in the bandwidth estimation. (a) HELLO packet is transmitted from sender to its neighbour, (b) HelloACK carry the timestamp Ts back to the sender.

In our algorithm, the parameter $S$ includes not only the size of the HELLO and HelloACK packets, but also the size of all packets exchanged between the node and its neighbour, including the MAC messages (RTS, CTS and ACK). Therefore, $S$ can be calculated as shown in Equation 2.

$$
S=R T S+C T S+\text { Hello }+ \text { ACK }+ \text { RTS }+ \text { CTS }+ \text { HelloAck }
$$

In the second step of the estimation of the local available bandwidth, each node calculates a weighted average of the most recent values of the Local Available Bandwidth $\left(\widehat{B W}_{\mathrm{av}}\right)$ in order to obtain a stable and yet responsive system of estimation (Equation 3).

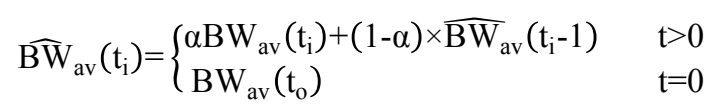

Where $\widehat{B W}_{a v}\left(t_{i}\right)$ is the new weighted average of the local available bandwidth, $B W_{a v}\left(\mathrm{t}_{\mathrm{i}}\right)$ is the actual measurement of the local available bandwidth in the time period $t_{i}, \widehat{B W}_{a v}\left(t_{i-1}\right)$ is the weighted average calculated in the previous time period $t_{i-1}$, and $B W_{a v}\left(\mathrm{t}_{\mathrm{o}}\right)$ represents the initial measurement of the local available bandwidth. We use $\alpha=0.8$, which were determined by a few empirical trials. This value of $\alpha$ allows the algorithm a fast reaction to changes of the network condition. A more detailed analysis of the algorithm for estimating the available bandwidth as well as details about its implementation, can be found in [34]. 


\subsubsection{Prediction of the consumed bandwidth along the path}

The second stage of the bandwidth estimation phase consists of estimating the consumed bandwith along the path to check if all nodes along the route could support the bandwidth requested by the source. In order to calculate this consumed bandwidth it is necessary to take into account the mutual interference between packets of the same flow. The method used to estimate of the intra-flow contention used in AQA-AODV is based on the parameter called Contention Count $(C C)$. Each intermediate node along a route calculate its $C C$ parameter according to the distance (number of hops) from itself to the source and destination nodes. Finally, the maximum value of the $C C s$ calculated along the route is sent to destination node. This method of calculation is based on the relation between the end-to-end throughput and the number of hops found in [32].

After estimating $C C$ in each intermediate node, the destination node can calculate the consumed bandwidth as shown in Equation 4. Where reqBW is the bandwidth requested by the source, $C C \max$ indicates the maximum value of the $C C$ parameters calculated in the intermediate nodes and $B W_{\text {consumed }}$ means the consumed bandwidth expected along the route if a flow is transmitted through path with data rate equal to reqBW.

$$
B W_{\text {consumed }}=C C_{\max } \times r e q B W
$$

In summary, when a destination node receives a $Q R R E Q$ packet, it calculates the $C C m a x$ and the $B W_{\text {consumed }}$ according to the Equation 4. Subsequently, the destination node compares the $B W_{\text {consumed }}$ with the last value of its local available bandwidth $\left(\widehat{B W}_{a v}\right)$, which has been calculated using the Equation 3. If $B W_{\text {consumed }}$ is less than $\widehat{B W}_{a v}$ in the destination node, it informs the source node that the transmission rate must be equal to the requested bandwidth (req $B W$ ). Otherwise, if the $\widehat{B W}_{a v}$ in the destination node is less than the value of $B W_{\text {consumed, }}$ the source must adjust its transmission rate to $\widehat{B W}_{a v} / C C_{\max }$.

As an example of the interaction of the algorithms used in the bandwidth estimation phase of our protocol, let us consider again the network described in the Fig. 2. Suppose that node 1 requests to transmit with a data rate of 120 Kbps $(r e q B W)$ to node 6 . In this case, the CCmax calculated will be 5, since the node 3 is at a distance of 2 hops from source node and of 3 hops from the destination. Therefore, the value of $B W_{\text {consumed }}$ calculated in the destination node will be $600 \mathrm{Kbps}(120 \mathrm{Kbps} \times 5)$. Assuming that the local available bandwidth $\left(\widehat{B W}_{a v}\right)$ in the node 6 is $400 \mathrm{Kbps}$, we can see that the consumed bandwidth ( $B W_{\text {consumed }}$ ) is higher than the $\widehat{B W}_{a v}$. This means that if the source node transmits to $120 \mathrm{Kbps}$ the destination could not support such rate. Therefore, the destination calculates a new rate according to its local available bandwidth. Then the destination node notifies the source that the transmission rate must be $80 \mathrm{Kbps}\left(\widehat{B W}_{a v} / C C_{\max }=400 \mathrm{Kbps} / 5\right)$. This data rate is lower than the rate of $100 \mathrm{Kbps}$ reported to the source in the previous example described in the section 4.1. This difference is due to the fact that we have introduced the estimation of the consumed bandwidth taking into account the mutual interference between packets of the same flow. A description about the integration of the bandwidth estimation phase in the route discovery process is detailed in the next section.

\subsection{Route Discovery in AQA-AODV}

In AQA-AODV, the route entry is created based on the application requirements. In our design, the application indicates in the request message the minimal bandwidth that must be guaranteed. If network cannot support this requirement, the application can adjust its data rate according to the value received from the network. For route discovery, if a source node requested a route to a destination node with specific bandwidth requirements, it broadcasts a $R R E Q$ packet with the QoS extension $(Q R R E Q)$ to its neighbour nodes (Fig. 5(a)). This packet includes a Session $I D$, which is used with the source address to uniquely identify each traffic flow. The Session $I D$ is generated by a counter which is incremented by a node each time it constructs a new QRREQ. The session id counter, as well as broadcast id counter used in AODV, is a separate counter that is maintained by each node.

When a node receives a $Q R R E Q$ packet, a reverse route entry is created with the Session ID, and the $Q R R E Q$ packet is rebroadcasted. This process continues until the $Q R R E Q$ packet reaches the destination node (Fig. 5(b)). In AODV, a RREP packet can be created by the destination node or an intermediate node with a "fresh enough" route to the destination. However, only the destination will be able to send the route reply packet (QRREP) in AQA-AODV. This will ensure that all nodes in the selected route satisfy the bandwidth constraints. When the destination node receives a $Q R R E Q$ packet, if it is a new request, a reverse route entry for the new session is created. Before sending the $Q R R E P$ to the source, local available bandwidth $\left(\widehat{B W}_{a v}\right)$ is checked and the consumed bandwidth along the path ( $\left.B W_{\text {consumed }}\right)$ is estimated. Finally, the $Q R R E P$ will be transmitted to the source with a modified header that includes the minimum value between the bandwidth requested by the source $($ req $B W)$ and the maximum bandwidth that all nodes along the route could support taking into account the intra-flow contention, (i.e. $\widehat{B W}_{a v} / C C_{\max }$ ) such as is described in section 
4.1.2. Once an intermediate node receives the $Q R R E P$ packet, it compares its local available bandwidth with the bandwidth indicated in the $Q R R E P$. If its local available bandwidth is lower, it replaces the value stored in the minbandwidth field of $Q R R E P$, with the value of its local available bandwidth. Otherwise, the node forwards the $Q R R E P$, see Fig. 5(c). This procedure will ensure that the source knows the minimum bandwidth along the path, which will be the maximum rate that it may transmit. Once the source node receives the QRREP packet, it adjusts its transmission rate according to the value of the field rate in $Q R R E P$ and then the transmission of the data packets is started, see Fig. $5(d)$.

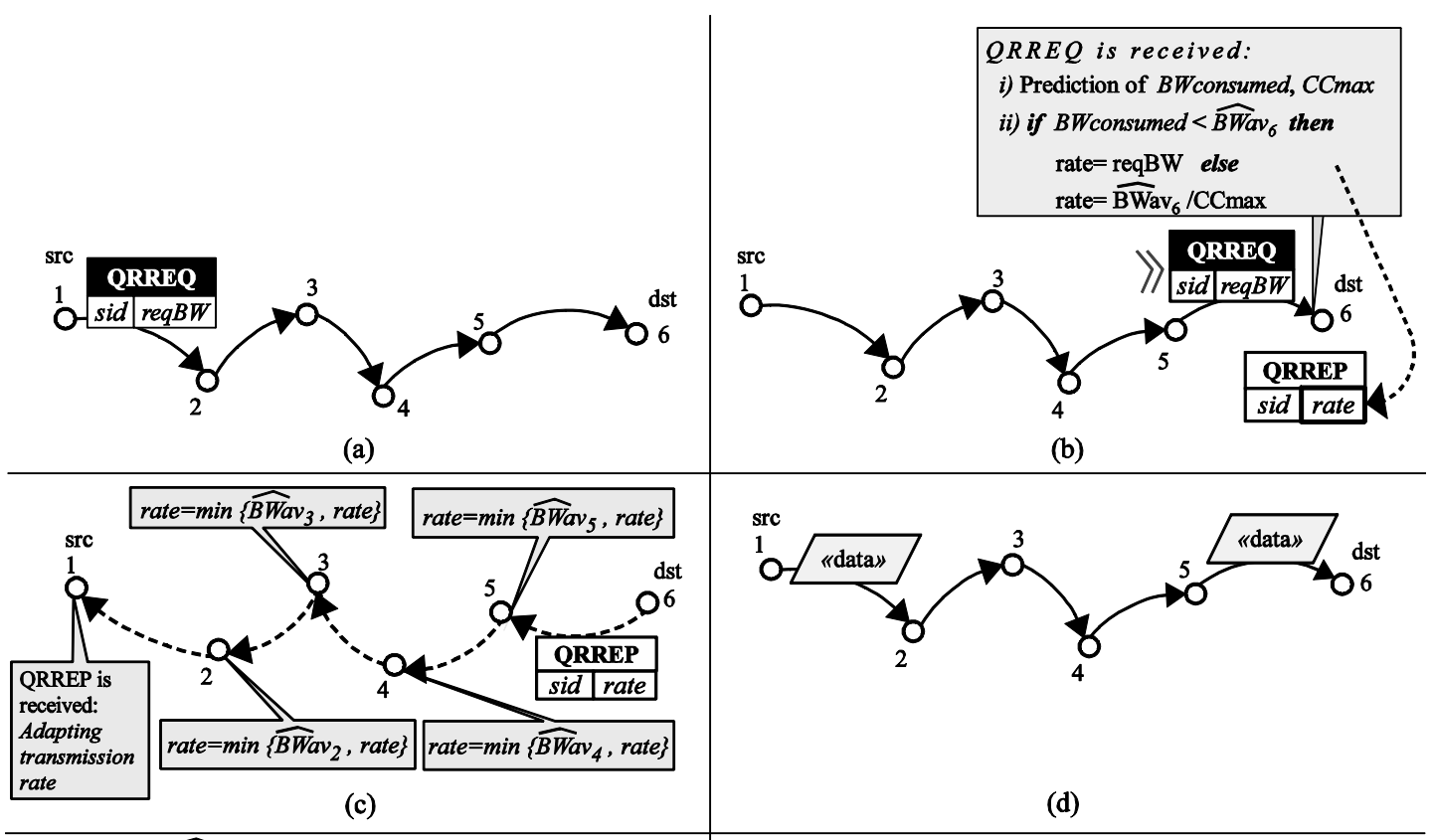

sid-Session ID, BW $\widehat{W v_{n}-}$ Local Available Bandwidth at node $n$, reqBW- Requested bandwidth, BWconsumed- Consumed bandwidth along the route, rate- Suggested Transmission Rate, QRREQ and QRREP- modified AODV packets with QoS extension (sid, rate, reqBW).

Fig. 5. Example of the route discovery phase in $A Q A-A O D V$.

\subsection{Route Recovery Mechanism in AQA-AODV}

Due to changes in topology caused by the mobility of the nodes and the condition of having a shared physical channel, the communications inside MANETs usually show frequent disruptions. For this reason, it is necessary to implement a route recovery mechanism. This mechanism not only has to re-establish the connections but also take into account the conditions of QoS that have been established during the route discovery phase.

The implemented route recovery mechanism in AQA-AODV detects the connection losses in a route when a host does not receive a HELLO message from a neighbour during an interval of time. The HELLO messages may not be received for three main reasons: 1) There is total connectivity but some of the HELLO messages are lost because of congestion; 2) The neighbour node is no longer available because it is out of transmission range and the node should look for a new path to the destination; and 3) The destination node is no longer available in the ad hoc network and the connection recovery is not possible.

Our route recovery mechanism perfectly works in any of the two previous cases in which connection recovery is possible. The functionality of the proposed route recovery mechanism is explained as follows (see Fig. 6).

During the route discovery phase (hereinafter called standard procedure of route discovery) each node from source to destination adds a register in its session cache list, see Fig. 6(a). Each register has a Session Identifier (sid) and an expiration time (Expiration Time) with the aim of erasing the old registers. Each time a node gets a data packet related to that session, it updates the expiration time of the registers, avoiding the elimination of the register and keeping the session alive, see Fig. 6(b). When some of the HELLO messages sent by an intermediate node are lost due to congestion, the adjacent nodes detects a link failure. They send an error message $(R E R R)$ to the source, including the affected session identifier, see Fig. 6(c). When source node receives the RERR message, it queries its session cache list using the session identifier received in the RERR message. Therefore, the source sends a $Q R R E Q$ message which includes the requested bandwidth, the actual data rate and the session identifier (sid). When the destination receives the $Q R R E Q$ message it checks if it has a register with the same sid as the one sent by the source in the QRREQ (Fig. $6(d))$. 
If it does have one, the destination node creates a $Q R R E P$ with the QoS parameters that had been negotiated during the initial route discovery phase. Moreover, it actives an immediate reply flag $(c=1$ immediate reply, $c=0$ standard reply) in the $Q R R E P$ message, which warns the intermediate nodes not to execute the standard procedure to verify the available bandwidth but send the $Q R R E P$ message directly to the next hop back to the source (see Fig. 6(e)). It is also possible that, due to the mobility of the nodes, the topology changes and the route to destination will be established through other nodes different from the ones used in the previously established route. In this case, when a new node in the route processes the $Q R R E Q$ message without finding a register associated to a session identifier (sid), it proceeds to generate a new sid. For this reason, when the $Q R R E Q$ message achieves the destination node it does not take into consideration the information of the previous session and it analyses the route request according to the standard procedure of route discovery (such as is described in the Section 4.2).

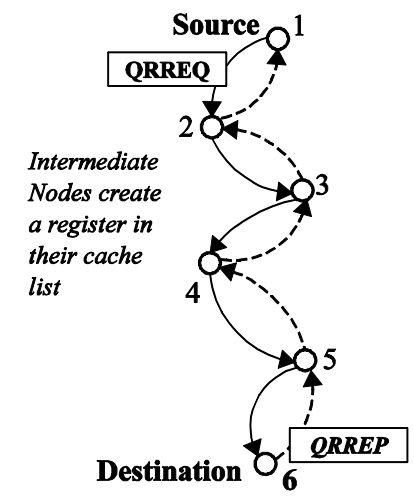

(a)

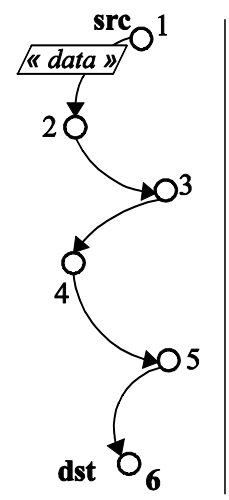

(b)

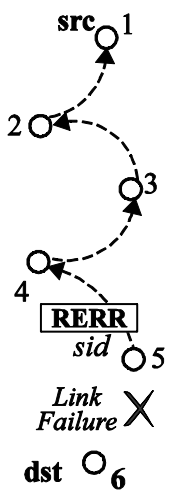

(c)

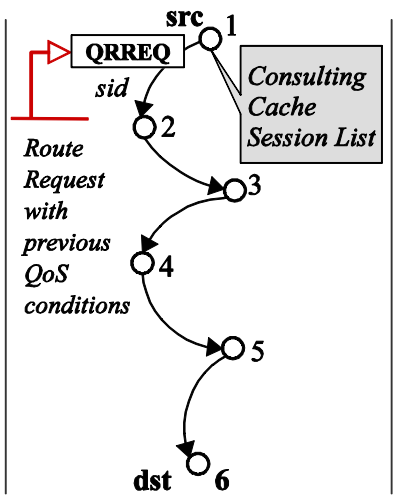

(d)

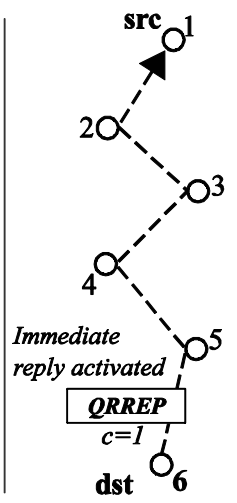

(e)

Fig. 6. Example of the route recovery mechanism of $A Q A-A O D V$.

We presented in [35] a more detailed description about the route recovery mechanism implemented in AQA-AODV. Similarly, in the Appendix 1 we present an algorithm in pseudocode that describes in a general way the procedures of the route-discovery and route-recovery process of AQA-AODV, previously seen.

In summary, AQA-AODV provides mechanisms not only for route discovery and route maintenance but also for estimating the available bandwidth. Moreover, it also provides a cross-layer feedback for sending information about the network state to application layer. Nevertheless, in a realistic scenario are necessary additional techniques to carry out the content adaptation taking into account the network conditions. Some possibilities include: $i$ ) semantic techniques, ii) having multiple versions for the same content and iii) scalable coding. Either option can be used to adapt the encoding rate by modifying characteristics of multimedia content, such as the resolution (dimensions of the video), the number of frames per second or the quality of the frames. Whereas the first option involves complex analysis of semantic information, the techniques of multiple versions require extra storage capacity since it will be necessary to store different copies of each video, with different quality levels, which is a non-scalable option. The third option allows us to have different levels of scalability in a single video stream. Therefore, it is not necessary to have multiple versions of the same content with different levels of quality, saving storage space. Thereby a wide range of terminals over heterogeneous networks can be served with a single version of the video. This is possible because the video stream will consist of several layers, each with different characteristics of quality. The number of layers that are sent to the client will depend on the state of network. This technique is called SVC (Scalable Video Coding) [2]. SVC has the advantage of scalability with a low computational cost, which is a very desirable feature especially when the service is accessed by a large number of users.

The combination of SVC with the available bandwidth estimation algorithms of AQA-AODV permits to build an adaptive system, which is able to adjust of the content quality to the transmission condition in order to avoid network congestion as well as further degradation of the quality of experience $(Q o E)$.

\section{ADAPTIVE SCALABLE VIDEO STREAMING}

The scalable video coding (H.264/SVC) generates different representations of the same video integrated within a same bit stream. A video encoded using the SVC standard has a layered structure where the layers correspond to different quality, spatial or temporal representations. A SVC video is composed of a base layer, which corresponds to the lowest representation, and one or more enhancement layers that increase the video quality when these are added to the base layer. The layered scheme of SVC provides higher robustness during video streaming over networks with continuous fluctuations of the bandwidth. SVC allows the sender to adapt the bit rate of the video traffic adding or removing SVC 
layers from the video stream based on the estimation of the available bandwidth (see Fig.7). Therefore, in order to adaptively control the bit rate of the video source, the adoption of cross-layer mechanisms in video streaming is required. Cross-layer solutions involves information exchange between the application layer, the network layer and the transport protocols to obtain optimal video data rates and routing policies [36]. In this paper, we propose the combined use of SVC and the cross-layer mechanisms included in AQA-AODV in order to build a framework for supporting adaptive video streaming that can significantly contribute to increasing the quality of video streaming services while the bandwidth efficiency is achieved.

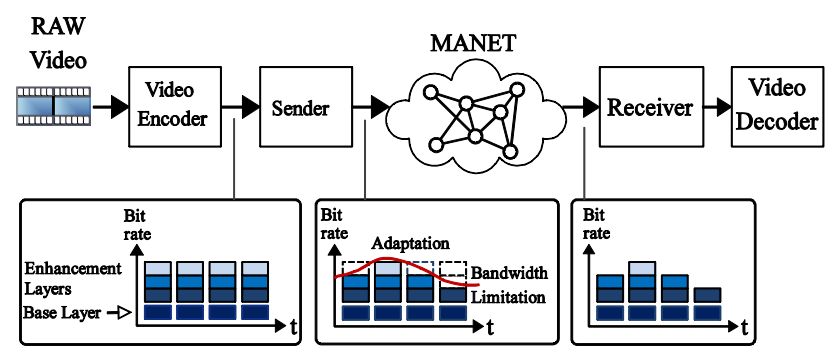

Fig. 7. Adaptive scalable video streaming in MANETs.

\subsection{H.264/SVC Scalable Video Coding}

In this section, we present a brief description of the main technical features of SVC, the scalable extension of the H.264/AVC standard. A more detailed explanation of the fundamentals of SVC can be found in the study of Schwarz et al. [37].

With H.264/SVC, the encoder produces a scalable bit-stream, which consists of a multiple layers. A base layer provides a basic video quality (e.g. low spatial or temporal resolution) and adding enhancement layers improves the quality (e.g. increases spatial resolution or frame rate). There are three modes of video scalability supported by SVC: temporal scalability, spatial scalability and quality scalability. When using temporal scalability, layers improve the frame rate. With spatial scalability, the base layer is coded at a low spatial resolution and enhancement layers give progressively higher spatial resolution. With quality scalability, the base layer contains a strongly compressed version of each picture, and enhancement layers incorporate more information to increase the SNR (Signal-Noise-Ratio) value. The H.264/SVC standard supports combined scalability, i.e. a scalable video can use any combination of the three types of scalabilities. For instance, Fig. 8 shows a SVC stream encoded with four temporal levels $\left\{T_{0}, T_{1}, T_{2}\right.$ and $\left.T_{3}\right\}$ and two quality levels $\left\{Q_{0}\right.$ and $\left.Q_{1}\right\}$. Thus, eight scalable layers are generated by combining these levels as listed in Fig. 8. The base layer consists of the lowest temporal resolution $\left(T_{0}\right)$ and the lowest quality level $Q_{0}$ (i.e. frames 1 and 8). In addition, an example of enhancement layer can be the layer consisting of the temporal layer $T_{2}$ and the quality level $Q_{1}$. This encoded video stream exploits the hierarchical prediction structure using B-pictures for enabling temporal scalability. Moreover, the coding structure of the quality scalability uses the key picture concept.

SVC Layers are identified using sequence of three identifiers: dependency identifier (DID), temporal identifier (TID) and quality identifier $(Q I D)$. These identifiers represent a point in the spatial, temporal and quality scalable dimensions, respectively. The values of DID, TID and $Q I D$ are also known as DTQ parameters. For instance, the base layer should be identified as $(0,0,0)$ and the enhancement layer consisting of the $T_{2}$ and $Q_{1}$ levels should be identified as $(0,2,1)$. The inspection of the $D T Q$ values permits to identify the data belonging to a specific enhancement layer. This fact is particularly important since this information may be identified and removed from the SVC encoded video, in order to reduce the bit rate.

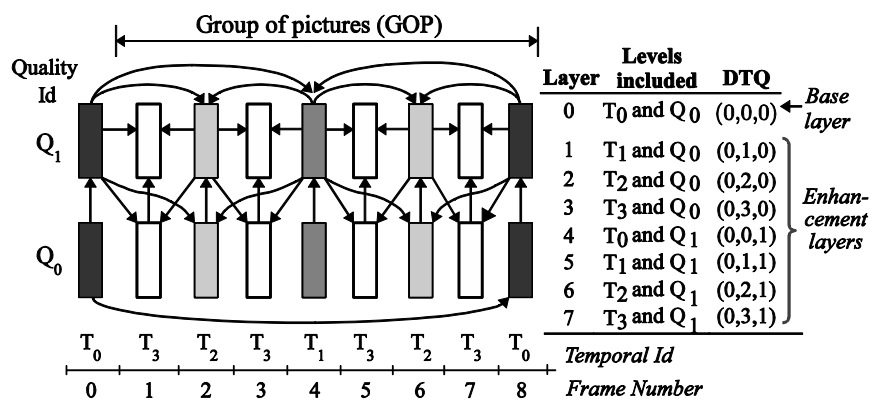

Fig. 8. Example of coding structure of a SVC stream with temporal and quality scalability 
In H.264/SVC, the codec is divided in two subsystems: the Video Coding Layer (VCL) and the Network Abstraction Layer (NAL). Basically, the VCL is in charge of the source video coding and the NAL is the interface between the encoder and the actual network protocol, which will be used to transmit the encoded bit-stream. In this work, we focus our attention on the NAL subsystem, since it provides the required information to identify the data relating to each layer. Nevertheless, the dependencies of the layers would be taken into account. Layers in SVC can be decoded independently but there is a logical dependency between them. This interdependency must be considered in order to obtain a correct decoding of the video. In the example shown in Fig. 8, the arrow lines represent dependencies between frames in a combined scalable stream. For instance, the frame 2 of the layer $T_{2} Q_{0}$ depends on the layer $T_{0} Q_{1}$ and the layer $T_{1} Q_{1}$. Because of these dependencies, discarding a quality layer from a reference frame (e.g. frame 2$)$ affects the quality of dependent frames (e.g. frames 1 and 3$)$.

\section{SIMULATIONS AND PERFORMANCE EVALUATION}

In this section, we investigate the performance of our proposed protocol and compare it with AODV and the implementation of QAODV conducted by Liu et al. in [17] through an extensive set of simulations. We take QAODV for performance comparison with AQA-AODV, because it is the closest protocol to AQA-AODV as compared with other QoS-aware protocols.

The objective of our simulation study is twofold: firstly, to evaluate the performance of our QoS-aware routing protocol by comparing it with the well-known AODV protocol and with a QoS routing protocol like QAODV. Secondly, we aim to demonstrate that our proposed solution is an effective system for providing video streaming services over MANETs.

\subsection{The Simulation Environment}

Network Simulator 2 (NS-2) [8] has been used to test the performance of our QoS-aware routing protocol. NS-2 contains the IEEE802.11 protocol in the MAC layer working in the distributed coordination function (DCF) mode with a channel data rate of 2 Mbps. The radio propagation model is Two Ray Ground and queue type is Drop Tail with maximum length of 50 packets.

The traffic flow used in the simulations consists of a video stream, which has been created by concatenating the wellknown test sequence SINTEL TRAILER [38] with a resolution of 1280x720 pixels (720p Format and 16:9 aspect ratio) to form a testing video of 2506 frames. The video sequence has been encoded according to H.264/SVC standard with two types of scalability: temporal and quality. As SVC codec, the JSVM codec was used [39]. All the values for the video related parameters are reported in Table 1.

Table 1. Video Parameters

\begin{tabular}{l|l}
\hline Parameters & Description/Value \\
\hline Original Video file & YUV format \\
Size & 2506 frames \\
Frame per second & 24 \\
Duration & $104.4 \mathrm{~s}$ \\
Encoded video file & H.264/SVC \\
Type of scalability & SNR (MGS) \\
B-Frames & Yes \\
GOP Size & 16 frames \\
\hline
\end{tabular}

The video sequence was encoded in five temporal layers (from $T_{0}$ to $T_{4}$ ). At the same time, we can add up to three extra levels of quality scalability (from $Q_{0}$ to $Q_{3}$ ) at each temporal level. For quality scalability, we use MGS (Medium Grain Quality Scalability) layers. The use of MGS layers for quality scalability allows source video to discard the data units from the enhancement layers without affecting the result bit-stream. Fig. 9(a) gives a graphical description of the bit rates obtained according to the temporal levels and the MGS layers. The labels on the bars indicates the layer id assigned by the SVC encoder. In total, we obtained twenty video layers (from $L_{0}$ to $L_{19}$ ) from the combination of sublayers $T_{i}$ and $Q_{j}$. The Y-axis in Fig. 9(a) indicates the bit rate associated to each layer. Depending of the number of transmitted layers, the output bit rate varies from $79.4 \mathrm{kbps}$ (sending Layer $L_{0}$ alone) to $775.7 \mathrm{Kbps}$ (sending Layers 0 to 19$)$. These values are aggregated, which means that to transmit Layer $L_{3}\left(T_{3} Q_{0}\right)$ we also have to transmit the dependent lower layers, i.e., $L_{0}, L_{1}$, and $L_{2}$. Therefore, the total bandwidth required would be of $202 \mathrm{Kbps}$.

Moreover, a rate-distortion analysis in terms of average Y-PSNR (PSNR for the luminance component in the YUV colour space) versus average bit rate was computed off-line (see Fig. 9(b)). The computation of the Y-PSNR curves were performed by stripping out the layers, measuring the average bit rate, decoding the resulting video, and 
computing the average Y-PSNR. Each of these curves represents a temporal layer and each point corresponds to a MGS layer (from $Q_{0}$ to $Q_{3}$ ). This figure descries the increase in the video quality (in terms of Y-PSNR) depending on the number of quality and temporal layers that make up the video.

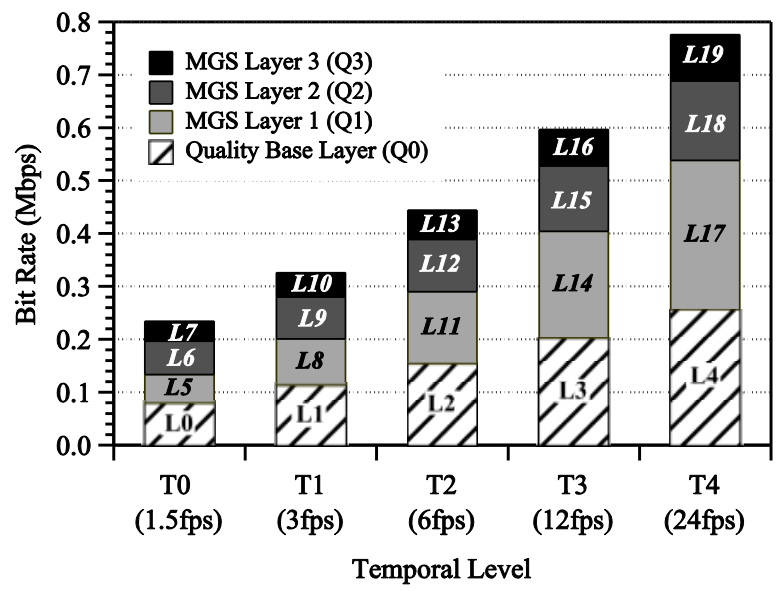

(a)

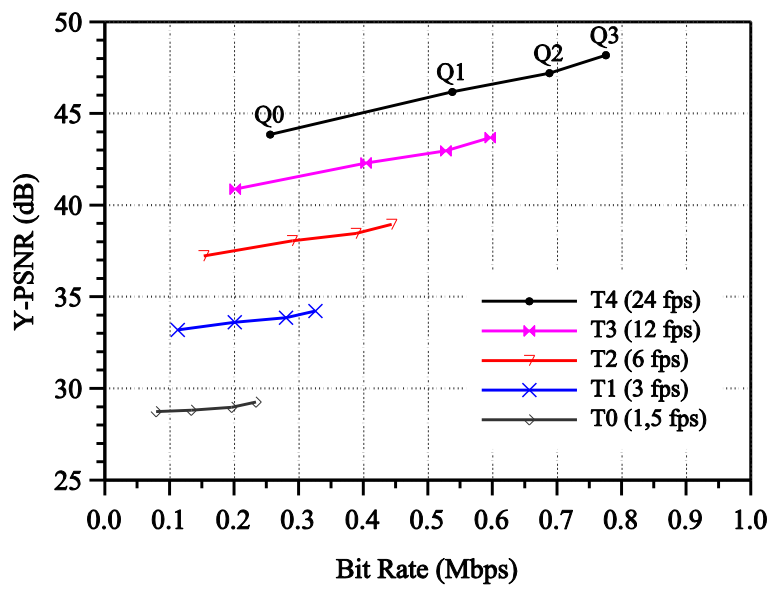

(b)

Fig. 9. (a) Description of the SVC layers contained in the video stream and (b) rate-distortion analysis of the SVC video stream.

\subsection{Simulation Scenarios}

We conducted two simulation studies to evaluate the performance of the proposed protocol. In the first simulation study, the effects of the network density over the bit rate adaptation are studied. The second simulation set aims to evaluate the influence of node movement on the performance of the adaptation algorithms of AQA-AODV and on the quality of video transmission.

In all simulated network scenarios, the video traffic is established between a random source-destination pair. In addition to video traffic, we also apply some CBR (Constant Bit Rate) flows as background traffic.

In order to simulate H264/SVC video transmission using NS-2, we have developed a video evaluation framework for adaptive video streaming, called SVCEval-RA [7], which is based on the well-accepted Evalvid platform [40] and its extended version for NS-2. In contrast to Evalvid, SVCEval-RA uses H.264/SVC encoding to support rate-adaptive video transfer. In addition, SVCEval-RA incorporates a tool set used to perform the assessment of video quality metrics, such as the PSNR (Peak Signal-to-Noise Ratio).

We evaluated the performance of AQA-AODV by measuring three parameters: end-to-end data packet delay, packet loss and the maximum throughput achieved along the route. In addition, we evaluated the rate of link failures (total number of link failures divided by the simulation duration) and the Connection Setup Latency (CSL), which is the latency incurred in establishing new connection from source to destination after the previous connection is lost (which includes route break detection time and recovery time). Moreover, we evaluated some parameters related to the quality of the transmitted video, such as the PSNR and the decodable frame rate. The decodable frame rate is an applicationlevel metric, which is defined as the ratio of the number of successfully decoded frames over the total number of frames.

We built and implemented in NS-2 a version of QAODV described in [17] with the aim of evaluating its performance and compare it with our protocol. Moreover, we plot the performance of AODV in the graphs in order to emphasize the performance improvements regarding the typical routing protocols. For each network scenario, we run the simulation for 10 times (with random scenarios with different seeds) to take average values in the measured performance metrics. The results are obtained with a confidence level of $95 \%$.

\subsection{Simulations Results}

\subsubsection{Simulations 1: network density analysis}

In the first network scenario, the performance of AQA-AODV was tested as function of the number of mobile nodes in the network. We model a mobile ad hoc network with 20,40, 60, 80, 100 and 120 mobile nodes placed randomly within a $1200 \mathrm{~m}$ x $500 \mathrm{~m}$ area. Simulations were run for 300 seconds and each data point represents an average of at 
least ten runs with identical traffic models, but different randomly generated mobility scenarios. Identical mobility and traffic scenarios are used across protocols. In order to avoid the spatial distribution change problem, the video stream starts being transmitted after 70 seconds of simulation. The detailed parameters of simulation scenarios are defined in Table 2. Initially the source requested a transmission rate of $0.350 \mathrm{Mbps}$, which be maintained constant when AODV and QAODV are used. However, using AQA-AODV, this transmission rate may be dynamically adjusted by the source because of the adaptive feedback scheme. In addition to the video flow, five flows of $10 \mathrm{Kbps}$ are introduced randomly as background traffic in the network. These traffic flows are CBR (Constant Bit Rate) over UDP.

Table 2. Relevant simulation parameters

\begin{tabular}{l|l}
\hline Parameters & Description/Value \\
\hline Wireless standard & $802.11 \mathrm{~b}$ \\
Wireless channel capacity & $2 \mathrm{Mbps}$ \\
Transmission range & $250 \mathrm{~m}$ \\
Interference range & $550 \mathrm{~m}$ \\
Total number of nodes & From 20 to 120 \\
Mobility model & Random waypoint model \\
Maximum speed & $10 \mathrm{~m} / \mathrm{s}$ \\
Pause time & $10 \mathrm{~s}$ \\
\hline
\end{tabular}

In Fig. 10 is depicted the variation of the total network throughput achieved using the three evaluated protocols. In detail, when the number of nodes is smaller than 40, AODV has the best throughput. This is because the routes established have enough bandwidth to support the transmission rate requested by the source, which allows video application to effectively transfer data packets. On Contrary, AQA-AODV has a transmission rate more conservative in order not to exceed the available bandwidth on the route. When the network has 50 nodes o more, the node density increases and the higher number of competing nodes also increase. This fact causes a decrease in the available bandwidth of the nodes. Under this network conditions AQA-AODV allows video source adapts its data rate transmitting only the layers that can be supported by the route. In any case, the throughput exceeds the effective available bandwidth, avoiding network congestion. In contrast, using a conventional technique of transmission in MANETs (such as AODV), the source does not know the available bandwidth and it injects packets to the network with a fixed rate of $0.350 \mathrm{Mbps}$ without adaptation. Established routes in networks with more than 60 nodes can support this data rate. Therefore, there is a significant increase in network congestion and packet loss as the number of nodes increases (see Fig. 11).

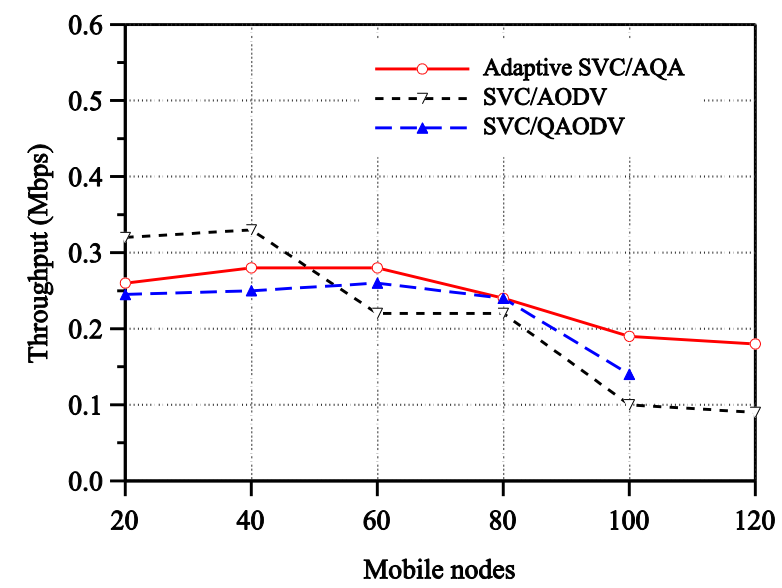

Fig. 10. Throughput achieved as a function of number of nodes. 


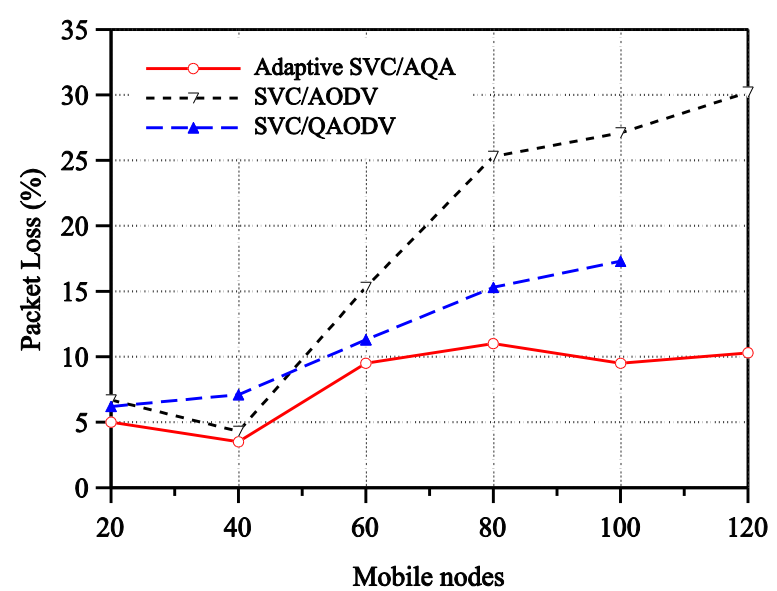

(a)

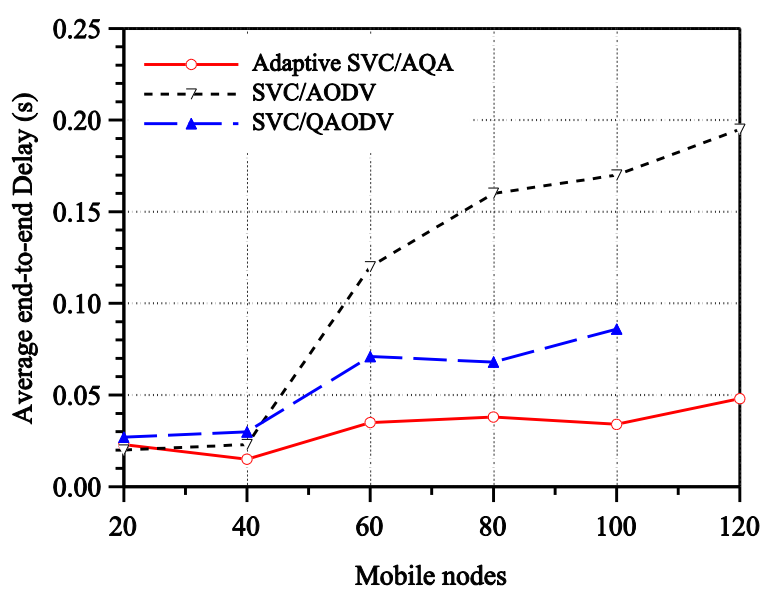

(b)

Fig. 11. (a) Packet Loss and (b) average end-to-end delay as a function of number of nodes.

Regarding QAODV, its admission control system accepts the traffic flow of $0.350 \mathrm{Mbps}$ in network scenarios with 100 or less nodes. In these scenarios, QAODV outperforms AODV in terms of throughput, average delay and packet loss. However, QAODV presents higher values of delay and dropped packets than AQA-AODV. This fact may be a consequence of the delay experienced during the search for a route that meets the requirements of bandwidth requested by the source. Moreover, in scenarios with more than 100 nodes, the traffic flow is rejected by the admission control system of QAODV since the route cannot support the requirements of the traffic flow.

The rate of link failures and the average values for the Connection Setup Latency (CSL) are presented in Fig. 12 (a and $b$ ). The data of the link failures are presented in relative terms (number of link failures over the simulation duration).

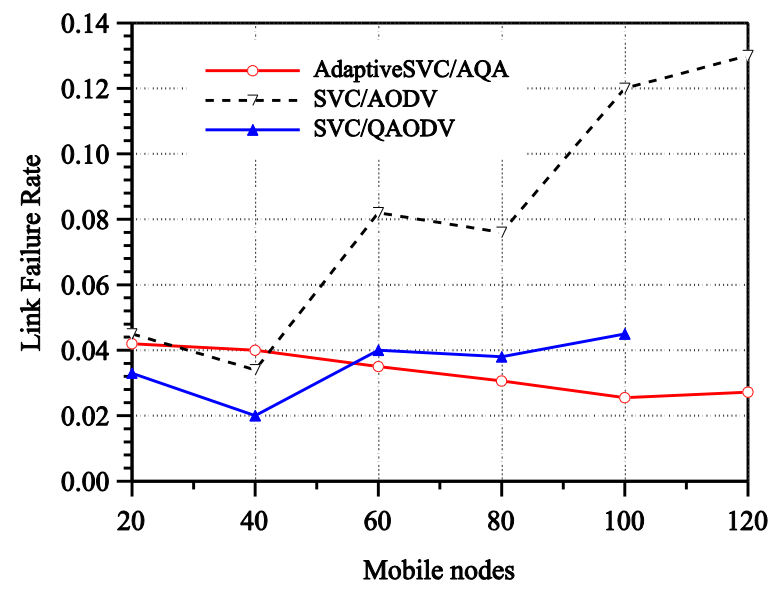

(a)

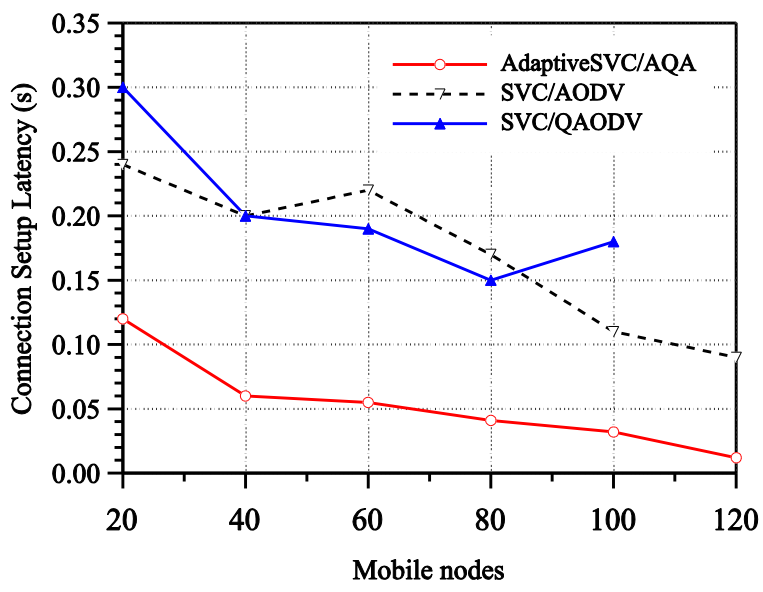

(b)

Fig. 12. (a) Rate of link failures and (b) average values of the Connection Setup Latency (CLS).

We notice that, the number of link failures drastically increases for AODV as the node density increases. These "supposed" link failures are caused by the loss of HELLO messages due to network congestion (such as was explained in Section 4.3). The results for CSL shows that the latency for re-establishing the routes has a descending trend for the three protocols. The reason is that as the number of mobile nodes increases, the ease of finding a new route also increases. In the case of QAODV, its route recovery process is less efficient due to the delay incurred in identifying a link failure and the larger latency re-establishing the routes. In contrast to AODV and QAODV, using AQA-AODV as routing protocol a more effective control of network congestion is obtained; consequently, only few link failures occurred. In addition, not only fewer link failures occurred, but also there was a decrease in the CLS, such as illustrated in Fig. 12(b). The route recovery mechanism of our approach makes faster the re-establishment of routes. Therefore, AQA-AODV is more prepared to support efficient video transmissions over network scenarios with high rate of link failures than other routing protocols. 
Concerning video transmission, Fig. 13(a) illustrates the decoded frame rate and Fig. 13(b) shows the average PSNR obtained during the network simulation. As observed in Fig. 13(a), the decodable frame rate is similar when the network has 60 nodes or less. Although some packets were lost in these network scenarios (mainly using AODV and QAODV), the robustness of the layered scheme of SVC provides an effective compensation. However, in scenarios where the network has more than 60 nodes, the decoded frame rate significantly decreases for the AODV and QAODV. In the case of AQA-AODV, this reduction is moderate and it is mainly caused by bit-rate adaptation performed by the video application, which sends only the packets belonging to the layers that can be supported by the route. The low rate of decoded frames of AODV is caused by the high rate of lost packets and the number of packets that have been discarded by SVCEval-RA tool after the play-out buffer deadline due to the high transmission delay. Regarding QAODV, although it allows the destination node to decode more frames than AODV, the high delay suffered during the link failures (CSL) makes the video quality is lower than that achieved with AQA-AODV.

As observed in Fig. 13(b), in the network scenarios with 60 or less nodes small differences between the three protocols, in terms of PSNR, are presented. In particular, AODV outperforms AQA-AODV in the network scenario with 20 nodes. As mentioned above, the reason is that in this scenario, the route established between source and destination node has an available bandwidth higher than the fixed transmission rate $(0.35 \mathrm{Mbps})$. Therefore, AODV allows video application to send video packets with this data rate without restrictions. Whereas using AQA-AODV only those SVC layers with aggregated bit-rate less than $0.35 \mathrm{Mbps}$ are transmitted. However, in scenarios with 40 nodes or more, the differences in quality between the videos transmitted by each protocol are more noticeable and AQA-AODV provides the highest video quality. For instance, in a network scenario of 100 nodes the differences are of $11 \mathrm{~dB}$ and $10 \mathrm{~dB}$ in relation to AODV and QAODV, respectively. These improvements are achieved because AQA-AODV maintains the video quality stable despite the increase in the number of nodes whereas AODV is affected by the high packet loss rate. With reference to QAODV, it cannot quickly find routes to destination due to the restrictions of its admission control scheme. This fact cause a latency that leads to a high packet loss rate and a low rate of decoded frames.

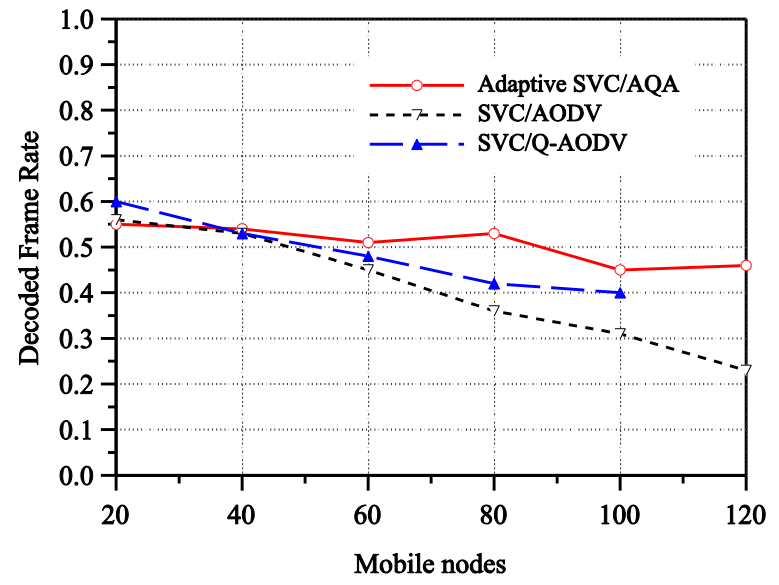

(a)

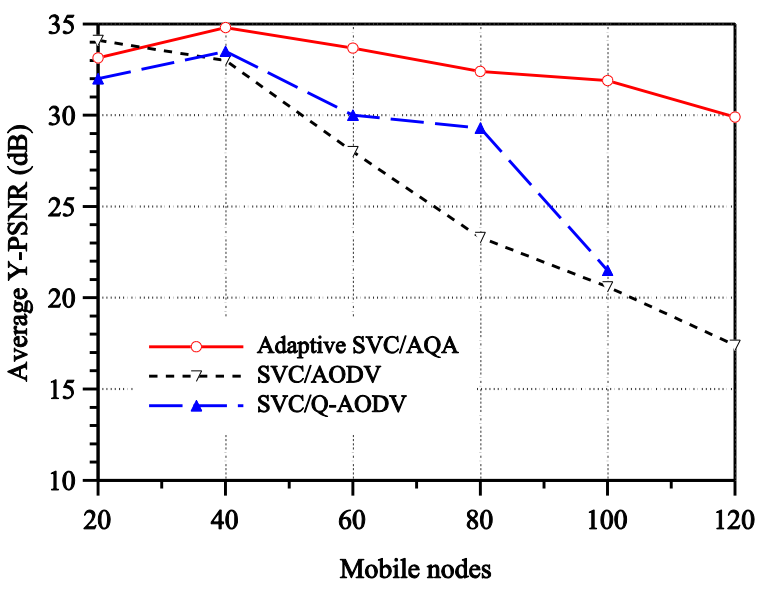

(b)

Fig. 13. (a) Decoded frame rate and (b) average Y-PSNR as function of the number of nodes.

In general terms, the results of this first set of simulation experiments demonstrate that the combination of the adaptive SVC scheme and the QoS mechanisms of AQA-AODV provides an efficient and reliable network-adaptive strategy. Even when the number of nodes in mobile network increases and the available bandwidth is more restrictive, AQAAODV enables a more stable video quality. This is due to the adaptive scheme presented in our proposed solution, which allows the traffic source to transmit only the SVC layers that can be efficiently supported by network. This fact provides better conditions to video streaming with an acceptable quality minimizing the pauses or video gaps caused by losses. Using AQA-AODV a feedback about the current network status is provided to the source application in order to set the layers that can be transmitted. Without this information, the video may not be adapted, causing congestion in the network and a large number of dropped packets.

\subsubsection{Simulations 2: mobility analysis}

In this simulation study, the network scenario has 30 nodes, which move in a rectangular area of $1000 \mathrm{~m} \times 300 \mathrm{~m}$ according to the random waypoint model. That is, the wireless node randomly selects a destination, moves in the direction of this location at a random speed, with a maximum speed of $5 \mathrm{~m} / \mathrm{s}$. Once the destination is reached, another random destination is targeted after a pause. With the aim of evaluating the influence of node movement on the quality of video transmission, we vary the pause time, which affects the relative speeds of the mobiles, from 0 to 120 seconds. A pause time of 0 seconds corresponds to the worst scenario because wireless nodes are all the time moving during the simulation. Transmission range for each node is $250 \mathrm{~m}$ and channel capacity is $2 \mathrm{Mbps}$. The traffic flow consists 
of a video stream of 2506 frames, such as was described in Section 6.1. Video source requests a bit rate of $775 \mathrm{Kbps}$, which correspond to the highest encoded bit rate of the video stream. As in the previous simulation experiments, five flows of $10 \mathrm{Kbps}$ were introduced as background traffic. This simulation scenario was intended to test the impact of the mobility of the nodes on the performance of AQA-AODV and on the video streaming quality. In order to evaluate the quality of the received video we have done several measurements, involving network and video metrics, such as packet loss rate, delay, decoded frame rate and Y-PSNR. These parameters are related to the objective quality of the reconstructed videos. The results of the video evaluation using AQA-AODV, QAODV and AODV are shown below.

Fig. 14 ( $\mathrm{a}$ and $\mathrm{b}$ ) show the results of our simulations in which the packet loss and average end to end delay are plotted versus the pause time. In terms of packet loss (Fig. 14 (a)), AQA-AODV shows an important improvement over AODV, which reaches very high packet losses as mobility of the nodes increases. More specifically, AQA-AODV outperforms AODV by about 40 percent at lower pause times (higher mobility) and 30 percent for higher pause times. The relative performance of AODV and AQA-AODV with respect to average end-to-end delay is similar to that with packet loss rate (Fig. 14 (b)). With AODV, the maximum average delay reaches $800 \mathrm{~ms}$ for a time pause of $0 \mathrm{~s}$ whereas using AQA-AODV always is maintained a delay below $97 \mathrm{~ms}$ (about 8 times lower than using AODV). The reason of the high values of the delay and packet loss for AODV is that the established routes between the sender and receiver nodes during the simulation time cannot support the transmission rate of $775 \mathrm{Kbps}$. Moreover, due to the lack of QoS mechanisms in AODV that allow the video application to adapt its data rate, a high level of traffic congestion is caused. With reference to QAODV, although this protocol shows a similar performance to AQA-AODV, only in very few cases the video sequence could be completely transmitted. For example, for pause times below $20 \mathrm{~s}$ the transmission of the video could not be started since the admission control of this protocol rejects the video traffic flow. For pause times above $20 \mathrm{~s}$, the transmission of the video packets is performed only for a short time interval, then QAODV rejects the traffic flow and the video transmission is cancelled. Hence, the points of the curve of QAODV represent measurements taken during the periods in which the video packets are streamed to the destination node. While the communication between source and destination is maintained, the obtained results with QAODV, in terms of packet loss and delay, seem to have a better behaviour, compared to AODV. We can also observe a slight increase of these metrics, compared to AQA-AODV, which may be caused by the delay of the route recovery mechanism of QAODV, which has a worse performance than the one of AQA-AODV such as demonstrated by measurements of the CLS (Connection Setup Latency) presented in Fig. 15 (b).

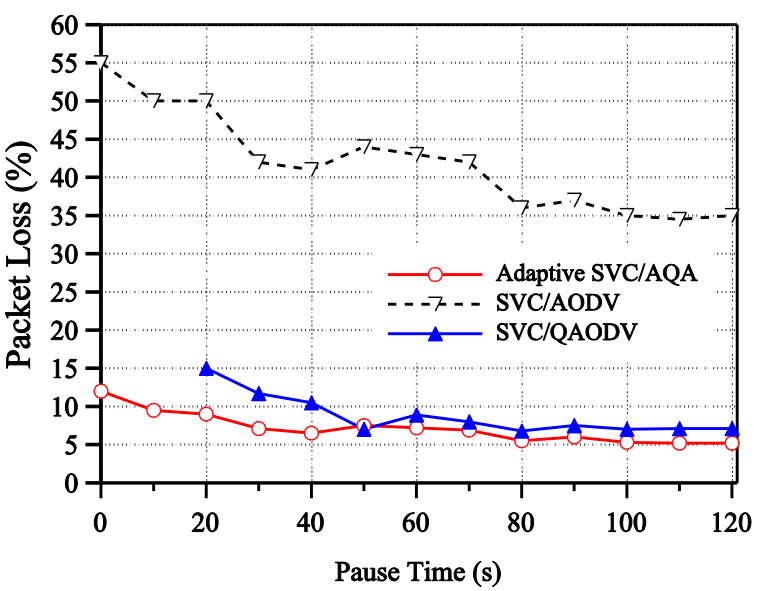

(a)

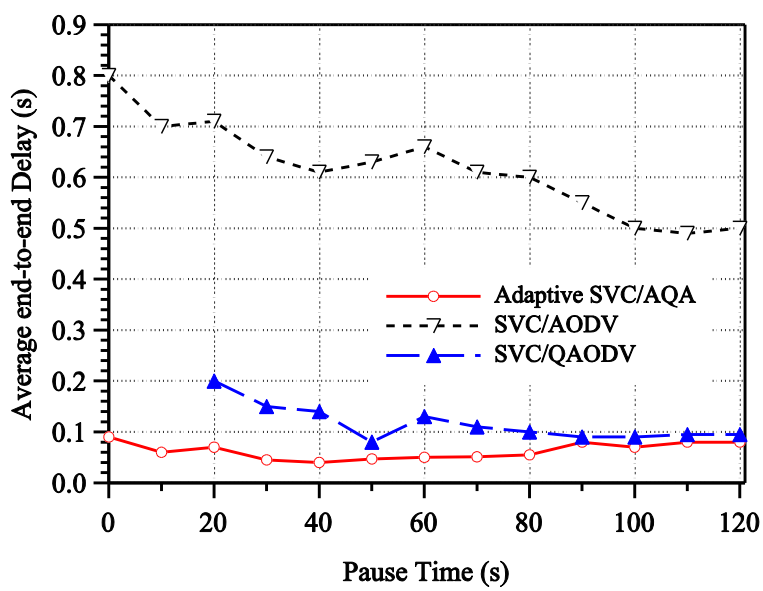

(b)

Fig. 14. (a) Packet Loss and (b) average end-to-end delay for a mobile scenario with different pause times

The problems caused by network congestion and the mobility of the nodes also can be observed in the frequencies of route break, mainly when AODV is used (see Fig. 15(a)). Each time a route breaks, there is some latency in the establishment of a new connection. This process includes time for route break detection, route discovery time and recovery time. Hence, packets get lost during this time interval, which could explain the growth of the packet loss in general terms for the three protocols as the mobility increases. Fig. 15 (a and b) show that both the number of link failures and the CSL of AQA-AODV is always lower than using AODV or QAODV. Comparing the three protocols we observe that there are fewer link failures and a shorter duration in AQA-AODV; consequently, there will be fewer gaps in the received video. The less duration of the re-establishment of the routes may be a consequence of the rapid mechanisms for the route recovery of AQA-AODV, such as the "immediate reply" strategy during the delivery of QRREP packets. It is important to note that the results for link failures are presented in relative terms (number of link failures per second) since some QAODV simulations have less duration. 


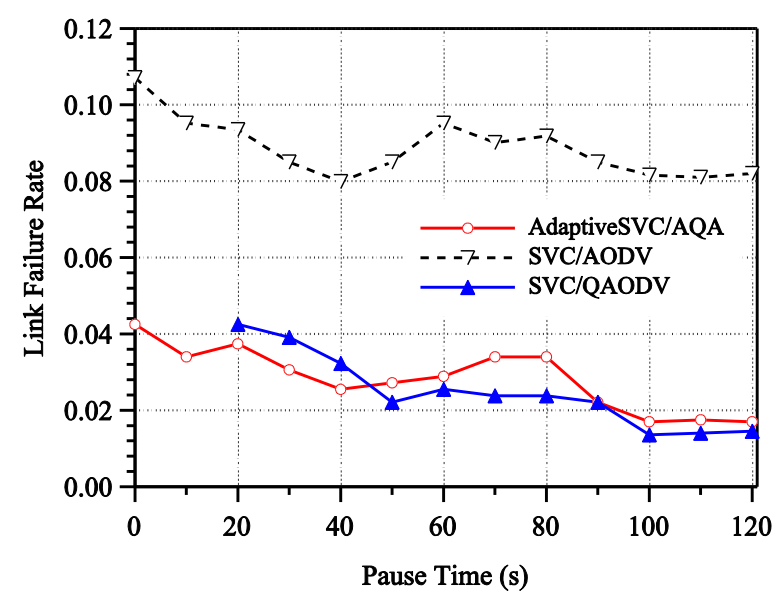

(a)

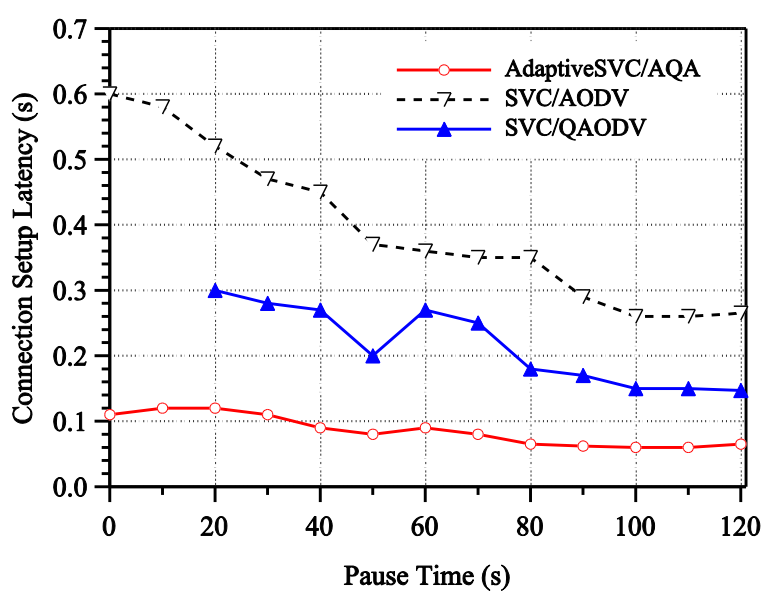

(b)

Fig. 15. (a) Number of link failures per second and (b) CSL (Connection Setup Latency) in mobile scenario with different pause times

As far as the video quality evaluation is concerned, Fig. 16 ( $a$ and $b$ ) report the decoded frame rate and the PSNR for the three protocols. The results in Fig. 16 show that under all mobility levels, AQA-AODV overall outperforms AODV and QAODV. Using AQA-AODV there was a high variation of the decoded frame rate with the increase of the mobility. For example, under low mobility conditions, the decoded frame rate is 0.25 and for high mobility is 0.55 , i.e., a difference of $54 \%$. This variation is mainly a consequence of the removal process of temporal layers from the video stream, performed by the video application in order to adapt the bit rate to the network state. On the other hand, the low values for the decoded frame rates using AODV are caused by both $i$ ) packet losses due to erroneous transmission over the wireless ad hoc network, and ii) packets discarded at the playout buffer because they were received too late at the destination node to be played out.

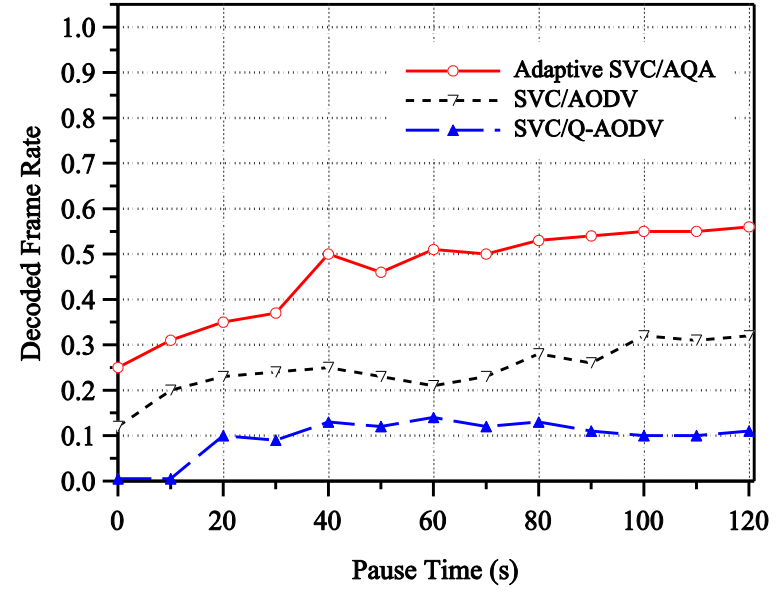

(a)

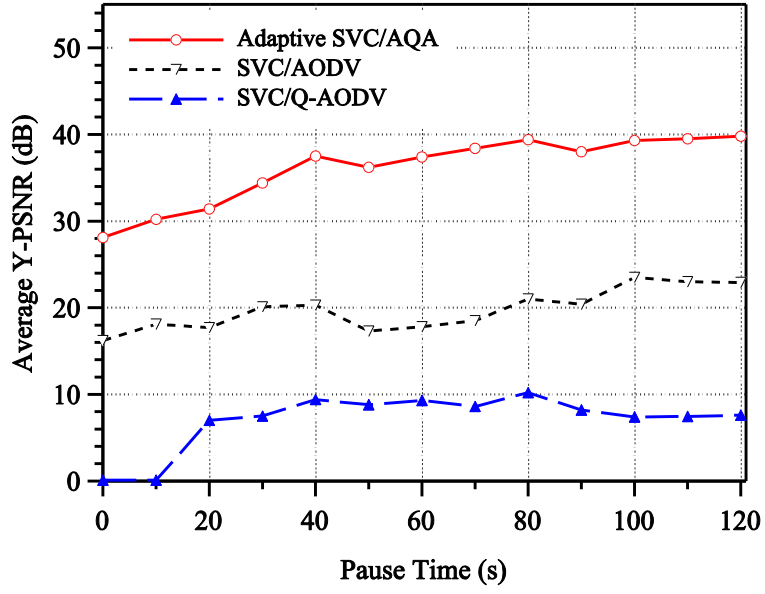

(b)

Fig. 16. Decoded Frame Rate and (b) average Y-PSNR as a function of the pause time

Finally, a significant improvement in the average PSNR is obtained using AQA-AODV, as can be seen in Fig. 16 (b). For the worst scenario (time pause of $0 \mathrm{~s}$ ), AQA-AODV improves the video quality in $11 \mathrm{~dB}$ in relation to AODV, whereas under low mobility (time pause above $80 \mathrm{~s}$ ) we obtained important improvements of $15 \mathrm{~dB}$ and $31 \mathrm{~dB}$, compared to AODV and QAODV, respectively. The poor results obtained by QAODV reveal its design based on a conservative admission control, though it can handle certain levels of QoS and avoids network congestion, it is not feasible for multimedia streaming in MANETs. In this case, it is much more efficient an adaptive system, which allow applications to adjust its data traffic to the available resources.

Even though the mobility conditions affect the performance of the three protocols, the combination of an adaptive feedback scheme and a fast re-routing algorithm allow AQA-AODV to minimize the impact of the mobility over the quality of the received video. Moreover, these algorithms also help avoid or reduce network congestion, minimizing the impact on the transmission of others traffic flows. 
In order to get a better insight into how quality degradation is distributed for a given video streaming depending on the available bandwidth, we focus again on a specific scenario. This sample scenario corresponds to that in which the pause time was set to 80 s. Fig. 17 reports the corresponding results for this sample scenario. The top graph in Fig. 17 shows the PSNR per frame of the video stream as a function of the frame index. The bottom graph illustrates the available bandwidth of the route between source and destination node. Both graphs are aligned to capture the variation of PSNR according to changes in the available bandwidth. Bandwidth curve also contains some negative spikes, which are caused by the link failures.

In Fig. 17 can be distinguished four intervals, such as indicated by the vertical markers on the graphs. In the first interval (i.e. between frames 0 and 826) the available bandwidth is above $900 \mathrm{Kbps}$; thus, all SVC layers are transmitted. This fact is because the bit rate required to send the highest layer and its dependent lower layers (i.e. 775 Kbps) can be supported by the route. For the next two intervals, the available bandwidth decreases to 400 and 200 Kbps respectively. This is due to the mobility of the nodes and multiple access interferences at certain regions of the ad hoc network. During these two intervals, using AODV, the video source continues sending packets at a fixed rate of $775 \mathrm{Kbps}$, which leads to network congestion. Thus, the high amount of dropped packet causes a significant decrease of PSNR. On the other hand, QAODV rejects the traffic flow since it cannot support the data rate requested by the video source; therefore, video streaming is cancelled. In contrast, AQA-AODV allows video application to decrease its bit rate transmitting only those layers that do not exceed the available bandwidth. For instance, in the second interval (from frame 826 to frame 1053) the layer $L_{14}\left(T_{3} Q_{1}\right)$ is transmitted whereas layer $L_{11}\left(T_{2} Q_{1}\right)$ and its dependent layers are transmitted in the third interval (frames 1054 to 1607). These layers have a temporal resolution of $12 \mathrm{fps}$ and $6 \mathrm{fps}$, respectively. Due to this fact, a large amount of frames (in the second and third intervals) has a lower PSNR than the frames in the first interval, where the frame rate was $24 \mathrm{fps}$. Subsequently, during the last interval the available bandwidth up to about $530 \mathrm{Kbps}$ and video source (with AQA-AODV) increases its bit rate transmitting the layer $L_{15}\left(T_{3} Q_{2}\right)$ and its dependent layers. In contrast, with AODV only a slight improvement is obtained due to the large frame losses of the above intervals.

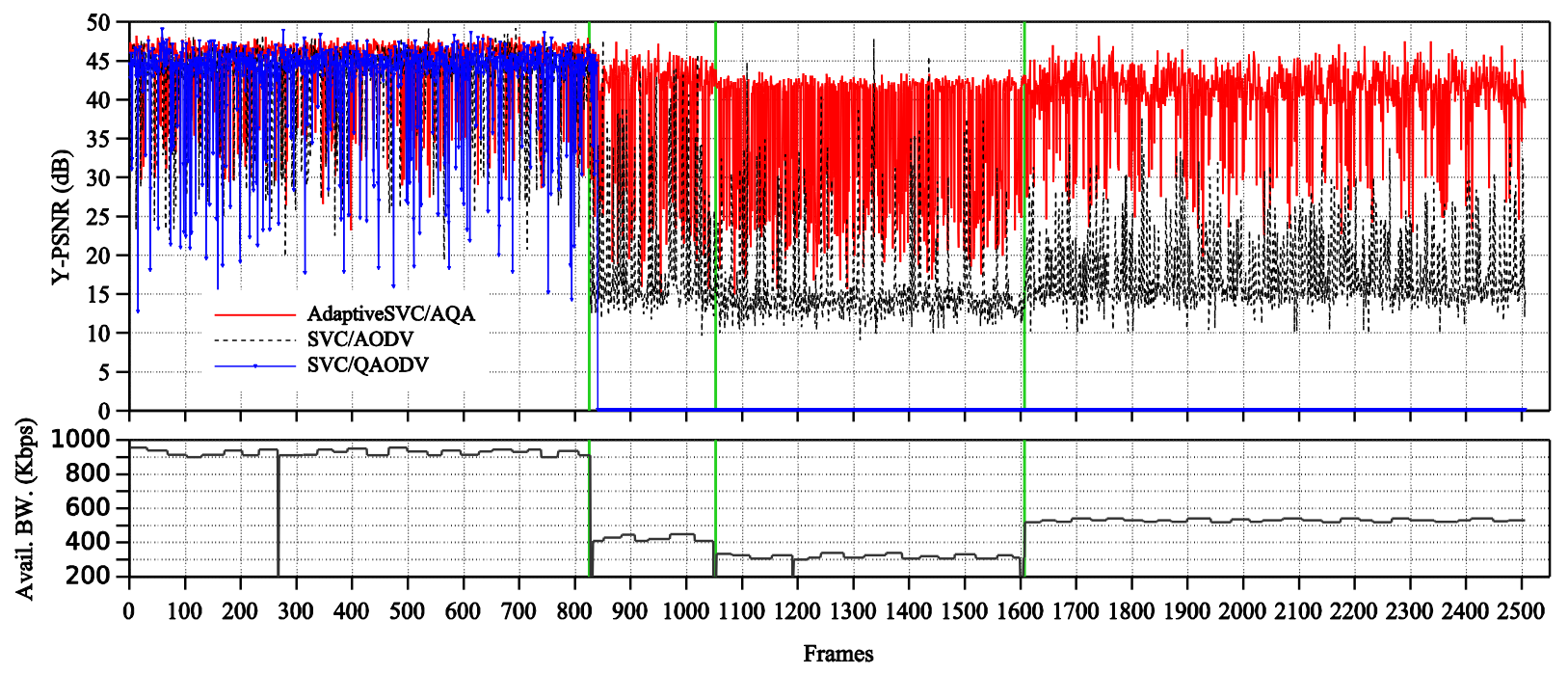

Fig. 17. Y-PSNR and Bandwidth Available as a function of the frame index (mobile topology, pause time $=80$ s.

In order to provide a subjective measure of the QoE, we make use of the Mean Opinion Score (MOS). In general, the MOS is a numerical indication of the quality of the media perceived by the end user, i.e., after transmission and decoding. Since MOS is a subjective metric, its assessment requires human interpretation. However, it is very much time consuming. For this reason, usually the MOS can be approximated by estimation from a corresponding objective metric, by means of a mapping table or a formula. In this case, we adopted the mapping defined in [40], which enables the conversion from PSNR to MOS as illustrated in Table 3.

Table 3. Possible PSNR to MOS conversion and impairment scale

\begin{tabular}{ccl}
\hline PSNR $[\mathrm{dB}]$ & MOS & \multicolumn{1}{c}{ Impairment } \\
\hline$>37$ & 5 (Excellent) & Imperceptible \\
$31-37$ & 4 (Good) & Perceptible, but not annoying \\
$25-31$ & 3 (Fair) & Slightly annoying \\
$20-25$ & 2 (Poor) & Annoying \\
$<20$ & 1 (Bad) & Very annoying \\
\hline
\end{tabular}


Fig. 18 shows the cumulative distribution function of the PSNR per frame in the considered scenario. The MOS levels, derived from PSNR values as described in Table 3, are also highlighted. From Fig. 18 we can observe how the variation of the network conditions affects the quality of the streamed video. In particular, with AQA-AODV the amount of frames with high PSNR values (i.e., those corresponding to the Excellent MOS level) is much larger than the corresponding amount with AODV and QAODV. On the other hand, with AODV, data loss has a significant effect on quality degradation such as evidenced by the high number of frames with low PSNR values (Bad to Poor MOS levels).

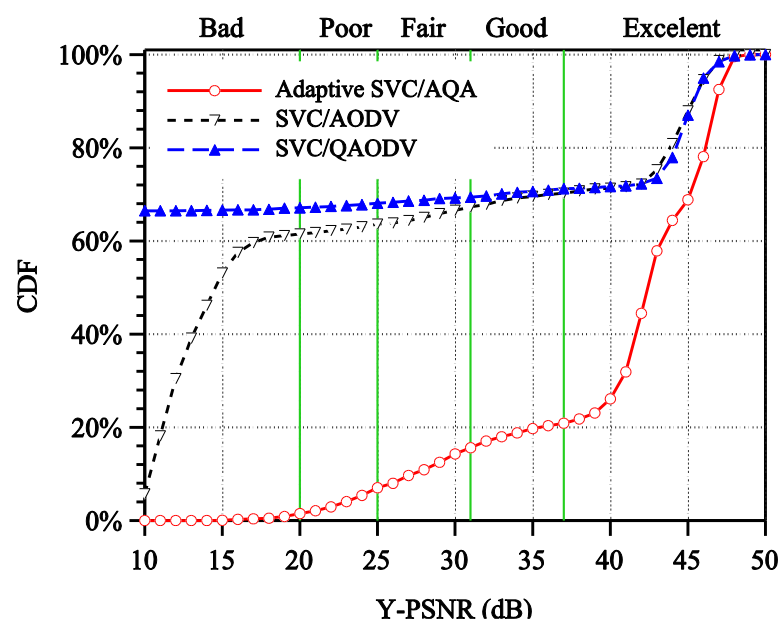

Fig. 18. Cumulative Distribution Function (CDF) of PSNR per frame (mobile topology, pause time=80 s)

\section{CONCLUSIONS}

A novel QoS-aware routing protocol (AQA-AODV) is proposed in this paper for carrying out time-sensitive communications over mobile ad hoc networks. We also proposed an adaptive method to exploit the layered scheme of SVC using the QoS parameters provided by AQA-AODV. This cross-layer method allows video source to adjust the bit rate of the video source adding or removing SVC layers from the original video stream based on the estimation of the available bandwidth. The integration of AQA-AODV and SVC provides a suitable system for supporting a network-adaptive strategy where video stream can be adapted avoiding network congestion and achieving a significantly improvement in the quality of the transmitted video. AQA-AODV incorporates a novel two-step process for estimating the available bandwidth of a route between source and destination node. In the first step, local bandwidth estimation is estimated in each node and, in the second step, it is performed a prediction of the consumed bandwidth that take into consideration the interference between packets of the same flow. In addition, we proposed a route recovery mechanism into AQA-AODV, which tries to re-establish connection to destination after a link failure, with the QoS conditions that had been negotiated during the initial route discovery phase.

A performance evaluation was conducted to assess our approach versus other QoS routing protocol, such as QAODV. Simulations show that the proposed cross-layer scheme of AQA-AODV could reduce significantly both the dropping rate and the end-to-end delay without impact the overall end-to-end throughput. Moreover, the results about CSL and link failures that our proposed mechanism is perfectly integrated into adaptive feedback scheme of AQA-AODV.

In terms of video transmission, the obtained results demonstrate that the combination of the layered scheme of SVC and the QoS mechanisms of AQA-AODV provides a realistic system for adaptive video streaming. The adaptive scheme presented in our protocol makes a more efficient use of the available bandwidth since it can provide feedback to the video application about the current network status in order to transmit only the SVC layers that can be efficiently supported by network. Without this network-adaptive strategy, the video may not be adapted, causing congestion in the network and a large number of dropped packets, which is much worse than transmitting video using low data rate. Consequently, the quality of the delivered videos has been significantly better than using AODV or QAODV.

The implementation of SVCEval-RA allowed us to use combined scalability (temporal and SNR) in the codification of the video sequences. Moreover, MGS scalability was used, instead of CGS (Coarse Granular Scalability), providing a better coding efficiency and a finer granularity during the adaptation process of the bit rate.

As future works, we plan to introduce further improvements to AQA-AODV, such as including support for end-toend delay during the route discovery phase. In addition, our future works include performing experiments using AQAAODV together with different network-adaptive protocols, such as TFRC and DCCP, assessing the quality of 
experience (QoE) of the user. Additionally, we intend to implement realistic video streaming services using DASH and AQA-AODV.

Some sample video sequences obtained during the simulation experiments can be displayed from the website http://www.comm.upv.es/aqa_aodv/aqa_aodv.html. Similarly, latest version of SVCEVal-RA framework and the source code of AQA-AODV are available for free download at [7] and [41], respectively.

\section{Appendix 1: Algorithm}

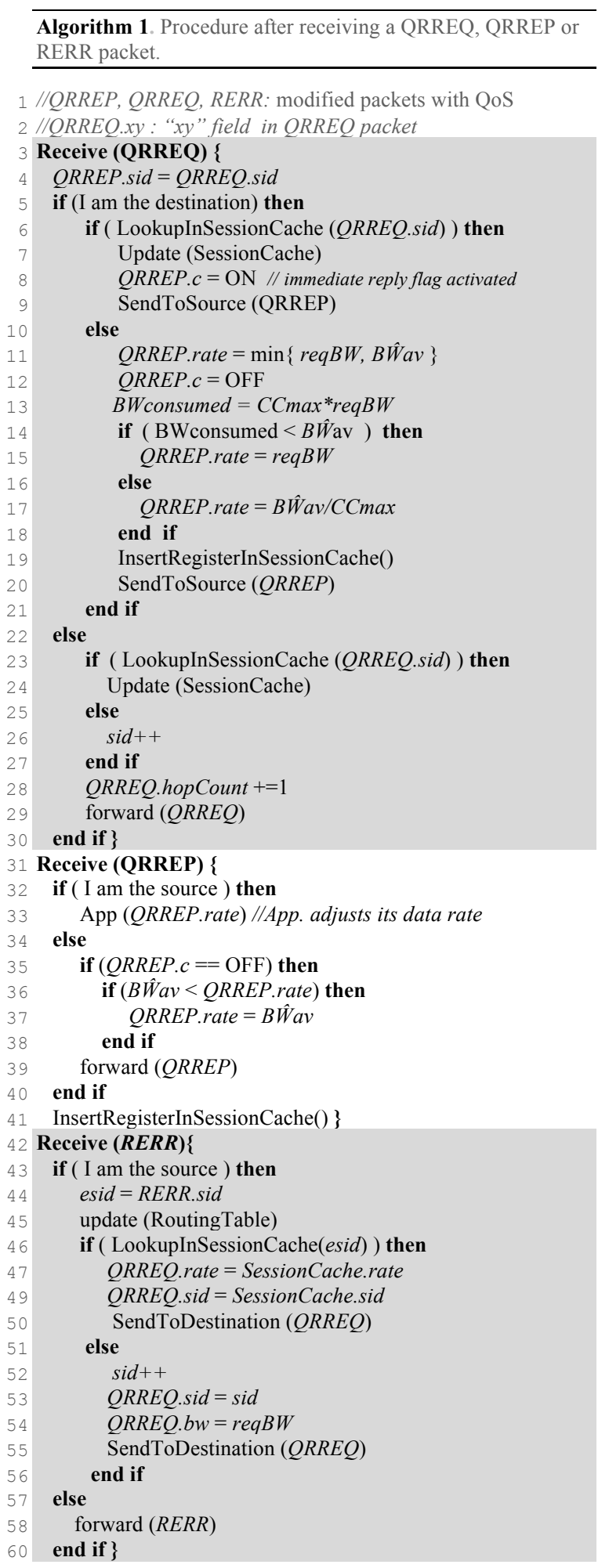




\section{REFERENCES}

[1] D. Tardioli, D. Sicignano, J.L. Villarroel, A wireless multi-hop protocol for real-time applications, Computer Communications. 55 (2015) 4-21. doi:10.1016/j.comcom.2014.08.012.

[2] ITU-T, Rec. H.264 \& ISO/IEC 14496-10 AVC. Advanced Video Coding for Generic Audiovisual Services, (2013).

[3] C. Perkins, E. Belding-Royer, S. Das, Ad hoc On-Demand Distance Vector (AODV) Routing, RFC 3561, (2003). http://tools.ietf.org/html/rfc3561.

[4] T.H. Clausen, C. Dearlove, J. Dean, C. Adjih, Generalized Mobile Ad Hoc Network (MANET) Packet/Message Format. IEFT RFC 5444, (2009). http://tools.ietf.org/html/rfc5444 (accessed May 18, 2013).

[5] C. Perkins, S. Ratliff, J. Dowdell, Dynamic MANET On-demand (AODVv2) Routing (IETF Internet Draft - 05), (2014). https://tools.ietf.org/html/draft-ietf-manet-aodvv2-05 (accessed May 16, 2013).

[6] M. Conti, S. Giordano, Mobile ad hoc networking: milestones, challenges, and new research directions, IEEE Communications Magazine. 52 (2014) 85-96. doi:10.1109/MCOM.2014.6710069.

[7] W. Castellanos, SVCEval-RA - An Evaluation Framework for Adaptive Scalable Video Streaming, SourceForge Project. (2014). http://sourceforge.net/projects/svceval-ra/ (accessed May 1, 2015).

[8] The Network Simulator (NS-2), (n.d.). http://www.isi.edu/nsnam/ns (accessed February 6, 2015).

[9] R. Geng, L. Guo, X. Wang, A new adaptive MAC protocol with QoS support based on IEEE 802.11 in ad hoc networks, Computers \& Electrical Engineering. 38 (2012) 582-590. doi:10.1016/j.compeleceng.2010.06.002.

[10] J. Hwang, T. Kim, J. So, H. Lim, A receiver-centric multi-channel MAC protocol for wireless networks, Computer Communications. 36 (2013) 431-444. doi:10.1016/j.comcom.2012.11.006.

[11] K. Kosek-Szott, M. Natkaniec, A.R. Pach, A new busy signal-based MAC protocol supporting QoS for ad-hoc networks with hidden nodes, Wireless Netw. 19 (2012) 1135-1153. doi:10.1007/s11276-012-0523-3.

[12] Y.-C. Tsai, S.-L. Su, An SINR-based routing and MAC design for QoS in wireless ad hoc networks, Wireless Netw. 21 (2014) 1141-1154. doi:10.1007/s11276-014-0840-9.

[13] M. Natkaniec, K. Kosek-Szott, S. Szott, G. Bianchi, A Survey of Medium Access Mechanisms for Providing QoS in Ad-Hoc Networks, IEEE Communications Surveys Tutorials. 15 (2013) 592-620. doi:10.1109/SURV.2012.060912.00004.

[14] S.-L. Su, Y.-C. Tsai, Y.-H. Yang, A Quality-of-Service Routing Protocol with Supplementary Cooperation for Wireless Ad Hoc Networks, Wireless Pers Commun. (2015) 1-19. doi:10.1007/s11277-015-2528-6.

[15] X. Zhen, Y. Wenzhong, Bandwidth-aware routing for TDMA-based mobile ad hoc networks, International Conference on Information Networking (ICOIN). (2013) 637-642. doi:10.1109/ICOIN.2013.6496701.

[16] C. Perkins, E. Belding-Royer, Quality of Service for Ad hoc On-Demand Distance Vector Routing (IETF Internet Draft), (2004). http://www.psg.com/ charliep/txt/aodvid/qos.txt (accessed May 23, 2012).

[17] L. Liu, L. Zhu, L. Lin, Q. Wu, Improvement of AODV Routing Protocol with QoS Support in Wireless Mesh Networks, Physics Procedia. 25 (2012) 1133-1140. doi:10.1016/j.phpro.2012.03.210.

[18] R. Mu, W. Zhang, QAODV: Improved Security Routing Protocol of AODV, Journal of Networks. 8 (2013) $2400-2405$. doi:10.4304/jnw.8.10.2400-2405.

[19] L. Ullah Khan, S.A. Mahmud, M.H. Zafar, G.M. Khan, H.S. Al-Raweshidy, M-AODV: Modified Ad Hoc On-demand distance vector routing scheme, in: 2014 9th International Symposium on Communication Systems, Networks Digital Signal Processing (CSNDSP), 2014: pp. 18-22. doi:10.1109/CSNDSP.2014.6923790.

[20] J. Zhou, L. Liu, Y. Deng, S. Huang, A QoS Routing Protocol with Bandwidth Allocation in Multichannel Ad Hoc Networks, Wireless Pers Commun. 75 (2014) 273-291. doi:10.1007/s11277-013-1362-y.

[21] M. Sanabani, R. Alsaqour, S. Kurkushi, A reverse and enhanced aodv routing protocol for manets, ARPN Journal of Engineering and Applied Sciences. 9 (2014) 153-159.

[22] S. Tabatabaei, M. Teshnehlab, S.J. Mirabedini, A New Routing Protocol to Increase Throughput in Mobile Ad Hoc Networks, Wireless Pers Commun. (2015) 1-14. doi:10.1007/s11277-015-2475-2.

[23] Y. Qin, L. Li, X. Zhong, Y. Yang, Y. Ye, Opportunistic routing with admission control in wireless ad hoc networks, Computer Communications. 55 (2015) 32-40. doi:10.1016/j.comcom.2014.09.007.

[24] L. Baccouche, J.D. Rekik, A QoS Architecture for Real-Time Transactions Guarantee in Mobile Ad Hoc Networks, Wireless Pers Commun. (2015) 1-22. doi:10.1007/s11277-015-2466-3.

[25] P. Jumnani, M. Zaveri, A Novel Cross-Layer Architecture for Video Streaming Over MANET, in: R. Maringanti, M. Tiwari, A. Arora (Eds.), Proceedings of Ninth International Conference on Wireless Communication and Sensor Networks, Springer India, 2014: pp. 11-25. http://link.springer.com/chapter/10.1007/978-81-322-1823-4_2 (accessed April 23, 2015).

[26] S. Pudlewski, N. Cen, Z. Guan, T. Melodia, Video Transmission Over Lossy Wireless Networks: A Cross-Layer Perspective, IEEE Journal of Selected Topics in Signal Processing. 9 (2015) 6-21. doi:10.1109/JSTSP.2014.2342202.

[27] M. Zhao, X. Gong, J. Liang, W. Wang, X. Que, S. Cheng, QoE-driven Cross-layer Optimization for Wireless Dynamic Adaptive Streaming of Scalable Videos over HTTP, IEEE Transactions on Circuits and Systems for Video Technology. PP (2014) 1-1. doi:10.1109/TCSVT.2014.2357094.

[28] C. Lal, V. Laxmi, M.S. Gaur, QoS-aware routing for transmission of H.264/SVC encoded video traffic over MANETs, in: 2013 19th Asia-Pacific Conference on Communications (APCC), 2013: pp. 104-109. doi:10.1109/APCC.2013.6765924.

[29] C. Lal, V. Laxmi, M.S. Gaur, S.-B. Ko, Bandwidth-aware routing and admission control for efficient video streaming over MANETs, Wireless Netw. 21 (2015) 95-114. doi:10.1007/s11276-014-0774-2.

[30] R. Choupani, S. Wong, M. Tolun, Multiple description coding for SNR scalable video transmission over unreliable networks, Multimed Tools Appl. 69 (2014) 843-858. doi:10.1007/s11042-012-1150-9.

[31] Y. Xu, C. Zhu, Multi-description multipath video streaming in wireless ad hoc networks, Signal Processing: Image Communication. 27 (2012) 836-848. doi:10.1016/j.image.2012.01.008.

[32] J. Li, C. Blake, D.S.J.D. Couto, H.I. Lee, R. Morris, Capacity of Ad Hoc wireless networks, in: Proceedings of the 7th Annual International Conference on Mobile Computing and Networking, ACM, New York, NY, USA, 2001: pp. 61-69. doi: $10.1145 / 381677.381684$. 
[33] K. Sanzgiri, I.D. Chakeres, E.M. Belding-Royer, Determining intra-flow contention along multihop paths in wireless networks, in: Proceedings of the First International Conference on Broadband Networks, IEEE Comput. Soc, 2004: pp. 611620. doi:10.1109/BROADNETS.2004.32.

[34] W. Castellanos, P. Acelas, P. Arce, J.C. Guerri, Evaluation of a QoS-Aware Protocol with Adaptive Feedback Scheme for Mobile Ad Hoc Networks, in: Springer Berlin Heidelberg, Las Palmas, Gran Canaria, 2009: pp. 120-127. doi:10.1007/978-3642-10625-5 8 .

[35] W. Castellanos, P. Arce, P. Acelas, J.C. Guerri, Route Recovery Algorithm for QoS-Aware Routing in MANETs, in: Springer Berlin Heidelberg, Bilbao. Spain, 2012: pp. 81-93. doi:10.1007/978-3-642-29479-2_7.

[36] C. Zhu, Y. Li, eds., Advanced Video Communications over Wireless Networks, CRC Press, Boca Raton, 2013. http://www.crcpress.com/product/isbn/9781439879986 (accessed April 14, 2015).

[37] H. Schwarz, D. Marpe, T. Wiegand, Overview of the Scalable Video Coding Extension of the H.264/AVC Standard, IEEE Transactions on Circuits and Systems for Video Technology. 17 (2007) 1103-1120. doi:10.1109/TCSVT.2007.905532.

[38] Xiph.Org Foundation, Video Test Media. Derf Collection, Video Test Media. Derf Collection. (n.d.). https://media.xiph.org/video/derf/.

[39] Moving Pictures Experts Group and ITU-T Video Coding Experts Group, H. 264/SVC reference software (JSVM 9.19.14) and Manual, (2011).

[40] J. Klaue, B. Rathke, A. Wolisz, Evalvid-A framework for video transmission and quality evaluation, in: Computer Performance Evaluation. Modelling Techniques and Tools, Springer, 2003: pp. 255-272. http://link.springer.com/chapter/10.1007/978-3-540-45232-4_16 (accessed May 13, 2014).

[41] W. Castellanos, AQA-AODV - A QoS routing protocol for MANETs, SourceForge Project. (2014). http://sourceforge.net/projects/aqa-aodv/ (accessed May 14, 2015). 